\title{
Integration of gene expression and brain-wide connectivity reveals the multiscale organization of mouse hippocampal networks
}

\author{
Michael S. Bienkowski ${ }^{1}$, Ian Bowman ${ }^{1}$, Monica Y. Song ${ }^{1}$, Lin Gou ${ }^{1}$, Tyler Ard ${ }^{1}$, K. Cotter $^{1}$, \\ Muye Zhu' ${ }^{1}$, Nora L. Benavidez ${ }^{1}$, Seita Yamashita ${ }^{1}$, Jaspar Abu-Jaber ${ }^{1}$, Sana Azam ${ }^{1}$, Darrick \\ Lo $^{1}$, Nicholas N. Foster ${ }^{1}$, Houri Hintiryan ${ }^{1}$, and Hong-Wei Dong ${ }^{1,2,3}$ \\ ${ }^{1}$ USC Stevens Neuroimaging and Informatics Institute, Center for Integrated Connectomics (CIC) \\ ${ }^{2}$ Department of Neurology, Keck School of Medicine of University of Southern California, Los \\ Angeles, CA, 90032, USA \\ ${ }^{3}$ Department of Physiology and Neuroscience, and Zilkha Neurogenetic Institute, Keck School of \\ Medicine of University of Southern California, Los Angeles, CA, 90032, USA
}

\section{Summary}

\begin{abstract}
Understanding the organization of the hippocampus is fundamental to understanding brain function related to learning, memory, emotions, and diseases like Alzheimer's disease. Physiological studies in humans and rodents suggest both structural and functional heterogeneity along the longitudinal axis of the hippocampus. Yet the recent discovery of discrete gene expression domains within the mouse hippocampus has provided the opportunity to re-evaluate hippocampal connectivity. To integrate mouse hippocampal gene expression and connectivity, we mapped the distribution of distinct gene expression patterns within mouse hippocampus and subiculum to create the Hippocampus Gene Expression Atlas (HGEA). Notably, novel subiculum gene expression patterns revealed a hidden laminar organization. Guided by the HGEA, we constructed the most detailed hippocampal connectome available using Mouse Connectome Project (www.MouseConnectome.org) tract tracing data. Our results define the hippocampus' multiscale network organization and demonstrate each subnetwork's unique brain-wide connectivity patterns.
\end{abstract}

\footnotetext{
Users may view, print, copy, and download text and data-mine the content in such documents, for the purposes of academic research, subject always to the full Conditions of use:http://www.nature.com/authors/editorial_policies/license.html\#terms

Correspondence should be addressed to H.W.D. (Hongwei.Dong@ini.usc.edu) or M.S.B. (Michael.Bienkowski@ini.usc.edu). Author Contributions

M.S.B. and H.W.D conceived, designed and managed the project. M.S.B. and H.W.D. wrote the manuscript. M.S.B. performed manual analysis of all raw image data including the gene expression and connectivity annotation, created the HGEA, and prepared figures for publication. I.B. led the informatics team, wrote the code for computational network analysis. M.S.B, I.B. and H.W.D. constructed the neural networks diagrams. M.S.B, M.Y.S, L.G., M.Z, and N.L.B. performed stereotaxic surgeries to generate anatomical connectivity data. K.C. and M.S.B. performed integrative analysis of HGEA annotation with DropViz data. T.A. created the 3-D HGEA atlas rendering along with online 3-D atlas viewer and supplementary videos. S.Y. managed the iConnectome website and created online informatics and visualization tools. J.A-J., S.A., and D.L. performed image processing for image data upload to the iConnectome viewer. N.L.B, N.N.F, and H.H. offered constructive guidance for the manuscript edits.

Competing Financial Interests

The authors declare no competing financial interests.
} 


\section{Keywords}

hippocampus; Alzheimer's disease; gene expression; connectome; atlas; network; tract-tracing

\section{Introduction}

The hippocampus is a highly specialized cortical structure located within the mammalian medial temporal lobe. Numerous studies over the last several decades have suggested structural and functional heterogeneity along the hippocampal longitudinal axis (dorsoventral axis in rodents, posterior/anterior axis in humans) ${ }^{1-6}$. Behavioral rodent studies suggest that dorsal hippocampus parts of the hippocampus are cognitive regions responsible for the spatial navigation/cognitive functions, whereas ventral hippocampus parts are affective regions related to emotional/affective behaviors and a core "limbic system" component of the classic Papez circuit ${ }^{1,4}$. However, gene expression and anatomical evidence suggests a more complicated organization within the hippocampus 7 . Decades of anatomical tract tracing studies in rats suggested a topographically-organized tripartite hippocampal projections from the entorhinal cortex (ENT) ${ }^{1,6}$; while connectivity between different hippocampal components (dentate gyrus (DG), CA3, CA1, and the subiculum (SUB) is organized as a continuous gradient with no definitive boundaries between the dorsal, intermediate, and ventral parts ${ }^{8}$.

Gene expression studies based on large-scale mRNA in situ hybridization data and modern RNAseq technologies have revealed a complex molecular heterogeneity of the mouse hippocampal formation ${ }^{1,9-14}$, which suggested the classic delineation of the hippocampus can be further refined. For example, recent mouse studies have revealed multiple discrete gene expression domains along the longitudinal axis with as many as 9 CA3 subregions, while the classic CA1 was divided into the dorsal, intermediate, and ventral domains ${ }^{1,9,10}$. However, no consensus has been made for how these hippocampal divisions should be refined and many of these studies focused only on "representative levels" and did not investigate the entire hippocampus (i.e., 1,9,10,15-17). Even less understood is how molecular heterogeneity of the hippocampus (transcriptomic data are mostly obtained in mouse) is correlated with its anatomical connectivity (which has been mostly conducted in rats). Although recent mouse connectomics approaches (i.e., ${ }^{18,19}$ ) have collected large scale connectivity data, the mouse hippocampus connectivity and gene expression data have not been systematically analyzed together.

To understand the relationship between hippocampal gene expression and anatomical connectivity, we have analyzed and annotated over 250 genes expressed throughout the entire hippocampus and subiculum, which were presented in the Allen Brain Atlas online gene expression database (www.Brain-map.org). The full rostrocaudal extent of each gene expression domain was mapped onto all corresponding coronal and sagittal Allen Reference Atlas (ARA) levels ${ }^{20}$ to create a Hippocampus Gene Expression Atlas (HGEA). Guided by the HGEA, we examined inputs/outputs of each HGEA domain and found that the gene expression boundaries delineated by the HGEA strongly aligned with anatomical connectivity patterns. Accordingly, we created the most complete hippocampal network 
wiring diagram to date, which will serve as a foundation for the functional dissection of the hippocampus.

\section{Results}

\section{General strategy for refining hippocampus parcellation based on a combination of molecular characteristics and connectivity}

To construct an accurate mouse hippocampus connectome, we refined the anatomical hippocampus delineation based on a combination of large-scale gene expression and connectivity data (Fig. 1, abbreviation list in Supplementary Table 1). First, we systematically analyzed and annotated spatial expression patterns of 258 hippocampalenriched genes selected from thousands of genes in the Allen Brain Atlas database (www.brain-map.org; annotation in Supplementary Table 2). By analyzing multiple gene expression distributions rather than a single gene, consensus patterns of similarities and differences become apparent (Fig. 1). Genes are sometimes expressed uniquely within the major hippocampal regions (i.e., CA3 vs. CA1) or in distinct combinations (i.e., DG and CA3, but not CA1). Other genes are more restricted within discrete parts of a region, thereby defining distinct domains which were otherwise indistinguishable. After determining a consensus gene expression-based hippocampal parcellation, we mapped the boundaries of gene expression domains in the DG, CA3, CA2, CA1, and SUB for both coronal and sagittal data to create the HGEA (coronal version Supplementary Fig. 1, sagittal version Supplementary Fig. 2, stereotaxic coordinate atlas versions available at www.MouseConnectome.org; see also Supplementary Videos 1-5).

To create the HGEA, we chose to manually analyze and delineate hippocampal boundaries due to inconsistent tissue sectioning across the coronal and sagittal Allen Brain Atlas database (see Supplementary Methods). The Allen Brain Atlas database is filled with thousands of mouse brains each identifying only one gene's expression pattern. Comparing different gene expression patterns across animals is complicated by differences in the oblique tissue sectioning of each brain (Supplementary Fig. 3). To account for this variability, we considered each part of each tissue section as independent (based on local anatomical landmarks) when manually mapping boundaries to the atlas. By applying this approach, gene expression mapping across all atlas levels allows for a more complete understanding of the shifting gene expression boundaries across the rostrocaudal axis.

Accordingly, we identified more than 20 molecular domains that are mapped onto the HGEA (see Fig. 1): 3 in the DG granule cell layer [dorsal (DGd), intermediate (DGi), and ventral domains (DGv)], 2 in the DG polymorph layer [dorsal (DGpod) and ventral domains (DGpov)], 5 in the CA3 [a rostral-dorsal tip (CA3dd), dorsal (CA3d), intermediate (CA3i), ventral (CA3v), and a ventral tip (CA3vv)], 4 in the CA1 [dorsal (CA1d), intermediate (CA1i), ventral (CA1v), and ventral tip (CA1vv)], 5 in the SUB (described in later section; sagittal gene expression data with sagittal HGEA atlas shown in Supplementary Fig. 4.). Overall, these results consolidated and refined earlier gene expression domain descriptions within DG, CA3, and CA1 ${ }^{1,9-14}$. In addition, we have compared the binary HGEA annotation to the more quantitative single-cell RNAseq database DropViz ${ }^{21}$ (www.DropViz.org) and found strong agreement between the two datasets. On average, 
$91.7 \pm 0.8 \%$ of HGEA annotated genes were found to have positive expression within related DropViz 'global clusters' (analysis available at www.MouseConnectome.org). A principal component analysis (PCA) of 248 HGEA genes found in the DropViz database generated a highly similar clustering pattern of hippocampal cell-types ('meta-cells') compared to PCA using all 32,307 DropViz genes (Supplementary Fig. 5). Finally, we calculated a dissimilarity matrix between the binary HGEA gene expression annotation and thresholded, binarized DropViz 'meta-cell' data of the 248 HGEA genes found in both datasets. The dissimilarity matrix shows strong similarities between specific HGEA regions and DropViz cell-types, supporting the idea that each HGEA region is composed of distinct combinations of individual cell-types (Supplementary Table 3). After creating the HGEA, we systematically examined each domain's input/output connectivity, constructed the hippocampus wiring diagram, and analyzed hippocampal network organization.

To present the anatomical data in a clear and detailed manner, case number (white) and injection site (color-coded with fluorescent labeling color) are listed at the bottom of each image (all case numbers, injection sites, and tracers are listed in Supplementary Table 5). The entire tissue series for all tract tracing data are openly available by searching the case number through the iConnectome viewer at our website (www.MouseConnectome.org). Furthermore, we have created several informatics and visualization tools to help navigating hippocampal networks (www.MouseConnectome.org/Analytics/page/matrix; Fig. 1).

\section{Delineating inputs/outputs of HGEA regions}

\section{Dentate gyrus gene expression subdomains and anatomical connectivity-}

The DG granule cell layer was divided into the dorsal, intermediate, and ventral domains (DGd, DGi, DGv) whereas the DG polymorph layer contains two distinct dorsal and ventral cell groups (DGpod vs. DGpov, Supplementary Fig. 6a). The DGpo is believed to contain a variety of neuronal cell-types including interneurons, that receive local inputs from the DG granule cells. The major DGpo projection neurons are the mossy cells, which are characterized by their longitudinally-broad bilateral projections to the inner-third of the DG molecular layer ${ }^{22}$. Both gene expression and connectivity data determined at least two discrete populations of mossy cells within the DGpod and DGpov, which display distinct projection patterns to innervate different segments of DG granular cells (Supplementary Fig. $6 b)$. DGpod neurons generate dense bilateral axonal projections to the DGd and DGi (DGpod $\rightarrow$ DGi/DGd), whereas DGpov neurons generate axonal projections to the DGv and DGi (DGpov $\rightarrow$ DGv/DGi). This connectivity pattern suggests that DG information can be integrated only in a ventral-to-dorsal direction (DGv $\rightarrow$ DGpov $\rightarrow \mathrm{DGi} \rightarrow \mathrm{DGpod} \rightarrow \mathrm{DGd}$ ), but DGd information may not directly reach the DGv (Fig. 2e).

Our DG connectivity analysis revealed differences between the DGd vs. DGi and DGv mossy fiber pathways. DGd mossy fibers follow a hairpin-shaped connectivity motif in which they first innervate the DGpo locally before travelling through the CA3 stratum lucidum and terminating on CA3 neurons along this path. DGd mossy fibers continue through CA3 up to $1 \mathrm{~mm}$ rostrally before turning back sharply to finally terminate in a topographically-organized CA2 subregions (Supplementary Fig. 6c,e). In contrast, DGi and 
DGv mossy fibers travel rostrally in a straight line to innervate CA3ic and CA3v/CA3vv, respectively (Fig. 2d, Supplementary Fig. 6d, f).

CA3, CA2, and CA1 gene expression subdomains and anatomical connectivity -The gene expression-defined CA3dd, CA3d, and CA3id resemble the morphologicallydefined 'CA3c', 'CA3b', 'CA3a' subregions first proposed by Lorente de No ${ }^{23}$, but other CA3 and CA1 gene expression domains do not appear to match this classic delineation. Within the CA1, gene expression patterns show regional differences that correspond to HGEA boundaries, although a more complex laminar pattern may exist (Fig. 2c). Finally, in agreement with Dong et al. ${ }^{9}$, some genes uniquely identify the ventral tip of the CA1v (CA1vv) as a distinct single layer domain (Supplementary Fig. 4b).

Our anterograde and retrograde coinjection data and multiple retrograde tracing data revealed that $\mathrm{CA} 3, \mathrm{CA} 2$, and CA1 domain connectivity patterns are highly consistent with other HGEA molecular domains (Fig. 2, Supplementary Fig. 7). CA3 gene expression patterns reveal highly restricted subdomains along the dorsal/ventral axis that were used to map HGEA delineations (Fig. 2a, b). After connectivity mapping, we found anatomical evidence to distinguish the more rostrally located dorsal CA3i (CA3id) from the caudal CA3i (CA3ic; Fig. 2a, b) as well as the rostral and caudal CA1d (CA1dr vs. CA1dc), but these subdivisions are not segregated in the gene expression-based HGEA (Supplementary Fig. 7). Using multiple retrograde tracer injections, we found retrograde labeling patterns that appear highly consistent with the boundaries outlined by the HGEA. Retrograde tracer injections into both CA 3 and CA1 subregions produced labeling patterns that respected HGEA borders suggesting that both CA3 associational connections and Schaffer collateral pathways are organized by CA3 gene expression domains (Fig. 2a, b). Additionally, anterograde and retrograde coinjections into individual CA3 subregions were found to label discrete sets of bilateral CA3 and CA1 subregions (Fig. 2c, Supplementary Fig. 8). CA3 domains project topographically and bilaterally to progressively more proximal CA1 domains (Fig. 2c, e). Generally, CA3dd projects to distal CA1d; CA3d and CA3id project to proximal CA1d, CA2, and CA1i; CA3ic projects to CA2, CA1i, and CA1v; CA3v projects to CA1v and SUBv; and CA3vv projects to CA1vv and SUBvv (note the dorsoventral limits of these terminal fields are highly restricted by HGEA boundaries rostrocaudally, see Supplementary Fig. 8).

The CA2 recently has received a great deal of attention due to the discovery of many specific gene expression markers that extends its classic borders (Supplementary Fig. 3e, f) ${ }^{24-26}$. Consistent with a recent monosynaptic rabies tracing study ${ }^{26}$, our anterograde tracing data shown here supports the DGd input to CA2 along the rostrocaudal axis (Supplementary Fig 6c,e). CA2 neurons receive DGd input along the rostrocaudal axis and then project topographically to the entire CA1 axis (except CA1vv). CA2 fibers within CA1 are primarily targeted to stratum oriens where a variety of inhibitory neuron cell types are distributed ${ }^{27}$. More rostral neurons in CA2 project to the CA1dr and CA1dc, whereas more caudal CA2 neurons project to the CA1i and CA1v.

Subiculum gene expression subdomains and anatomical connectivity-In the SUB, we found that gene expression patterns subdivided the pyramidal layer into 4 layers, 
although the layer distribution is non-uniform (Fig. 3a-c, Supplementary Figs. 1, 2, 4d). Similar to cerebral cortex parcellation, we defined 5 SUB subdivisions based on the unique combination and distribution of their gene expression layers. First, a 2-layered region, which is located within the "classic" dorsal subiculum (SUBd), contains gene expression layers 1 and 4 (red and yellow in HGEA, respectively). At both the dorsal and ventral ends of this domain, layer 4 forms a thicker, bulb-like shape which distinguishes the dorsal and ventral parts of the SUBd (SUBdd vs. SUBdv, Fig. 3c). Next, a 2-layered region containing gene expression layers 3 and 4 is located between the SUBdd and CA1d in a region that was first identified in the mouse by Lorente de Nó as the prosubiculum (ProSUB), although its presence in the mouse has remained controversial ${ }^{23,28}$. Furthermore, a 3-layered subdivision comprising gene expression layers 2,3 , and 4 is comparable to the classic 'ventral' subiculum (SUBv, see Fig. 3b). Finally, we also distinguished the ventral tip of SUBv (SUBvv) where layer 4 thickens and layer 2 thins before curving to wrap around the other layers.

Our anatomical tracing data shows that SUB connectivity is reflected by both the gene expression laminar organization and subregions outlined by the HGEA (Fig. 3a, b, Supplementary Movie 6). In the SUBv and SUBvv, layers 2 and 4 mediate distinct connectivity pathways with CA1 (Fig. 3d), while layer 4 also contains thalamic-projecting neurons (Fig. 3e). Each CA1 domain targets discrete combinations of SUB domains although CA1d organization is particularly complex (Fig. 3f). CA1d output to the SUBdd and ProSUB is organized rostrocaudally, whereas CA3 input to CA1d is organized along the proximo-distal axis (Supplementary Fig. 7). Overall, SUB gene expression layer connectivity is organized similar to the isocortex: SUB layer 2 primarily mediates intrahippocampal connectivity similar to the cortico-cortical connectivity mediated by isocortex layers 2 and 3; SUB layers 1 and 3 mediate different corticofugal pathways similar to isocortex layer 5; and SUB layer 4 contains thalamic-projecting neurons similar to isocortex layer 6 (Fig. 3g).

\section{Multi-scale network organization of hippocampus connectivity}

Using our anatomical tracer data, we manually annotated the inputs and outputs of all HGEA molecular domains to construct the hippocampus wiring diagram (Fig. 4a, Supplementary Table 5). To further understand the network organization of hippocampus connectivity, we first annotated and weighted all intrahippocampal connections between the HGEA subregions (Supplementary Table 6) and constructed a weighted, directed intrahippocampal connectivity matrix (Fig. 4b). Using this data, we determined the intrahippocampal subnetworks by running 1000 trials of a Louvain community detection algorithm at multiple gamma values (every 0.1 value between 0.1 and 20.0; 2,000,000 total iterations) and then calculated a Mean Partition Similarity (MPS) metric at each gamma value to determine the most representative consensus network partition. Gamma values with MPS peaks were observed at $0.15,1.36$, and 2.04 (see graph in Fig. 4b). Using these gamma values, we produced a schematic network graph and a reordered multiscale connectivity matrix featuring clustered network modules at gamma 2.04 as a subset of modules at gamma 1.36, which itself is a subset of modular networks at gamma 0.15 (Fig. 4b). The results show that at gamma 0.15 the hippocampus is clearly divided into a 'dorsal' and 'ventral' 
hippocampus (large red and blue outlined boxes in Fig. 4b matrix and wiring schematic). At gamma 1.36, the dorsal hippocampus is divided into four modular subnetworks ([DGd, DGi, DGpod], [CA3dd, CA3d, CA3id, CA3ic], [CA1dr, SUBdd], and [CA1dc, CA2, ProSUB]) and the ventral hippocampus is divided into three modular subnetworks ([DGv, DGpov], [CA3v, CA1v, CA1i, SUBv, SUBdv], and [CA3vv, CA1vv, SUBvv]) shown by shaded colored boxes. Finally, at gamma 2.04, the only distinction from the network partition at gamma 1.36 is that the CA3dd and CA3d splits from the CA3id and CA3ic (colored outlined boxes within shading in matrix and colored circles in schematic). Overall, our intrahippocampal analysis shows that the hippocampus is a multiscale structure that is organized as modular hierarchical subnetworks.

After defining the intrahippocampal subnetwork organization, we determined how each intrahippocampal subnetwork is connected to other brain regions as brain-wide neural networks. Using the annotated data in Supplementary Table 5, we ran the Louvain community detection algorithm using the same parameters as described for the intrahippocampal analysis. For this dataset, we found the highest MPS peak at gamma 9.74 with a corresponding consensus partition featuring 46 different communities. Based on these 46 different communities, we manually arranged each community into five subnetworks based on extrinsic connectivity similarity and constructed their network graphs (Fig. 4c, d). The results show the intrahippocampal subnetworks (from Fig. 4b) differentially innervate larger brain networks outside the hippocampus in a coordinated manner. The DG and CA3 regions contribute relatively little to extrahippocampal connectivity, but instead appear to integrate information from multiple intrahippocampal subnetworks across the hippocampal axis (Fig. 4c). In contrast, the CA1/CA2 and SUB regions are organized into three relatively distinct global networks (Fig. 4d). Next, we describe unique detailed features these three extrahippocampal networks.

\section{Detailed connections of the three brain-wide extrahippocampal networks}

The CA1dr-SUBdd/SUBdv network-Both SUBdd and SUBdv layer 1 neurons project to complementary areas within the ventral retrosplenial cortex (RSPv), postsubiculum (POST), presubiculum (PRE), and parasubiculum (PAR), and medial mammillary nucleus (MM), while SUBdd/SUBdv layer 4 neurons project to the anterodorsal (AD), anteroventral thalamic nuclei (AV), and reuniens thalamic nucleus (RE; Fig. 5a-d). The POST, PRE, and PAR generate dense projections through the fornix to the lateral mammillary nucleus (LM), which in turn sends massive bidirectional connections to the AD and AV. In parallel, both the SUBdd and SUBdv send dense projections to the laterodorsal thalamic nucleus (LD), which has robust connectivity with the visual (VIS), retrosplenial (RSP), and POST/PRE/PAR cortical areas (Fig. 5b). Fig 5e shows the overall connectivity of SUBdd/ SUBdv with visual and spatial brain areas (see Discussion).

The CA2/CA1dc-ProSUB and CA1i/CA1v-SUBv networks-According to our intrahippocampal network analysis, the CA2/ProSUB and SUBv receive distinct, but partially overlapping, intrahippocampal input: the ProSUB receives primary input from the "dorsal" hippocampus (primarily CA1dc), while the SUBv receives input from the "ventral" hippocampus (CA1i/CA1v). However, ProSUB and SUBv extrahippocampal connections 
are highly similar relative to the output of other CA1/SUB domains. Ultimately, our analysis groups the CA1i/CA1v/SUBv and the CA2/CA1dc/ProSUB as one broad brain-wide network with the medial prefrontal cortex (MPF), amygdala, nucleus accumbens (ACB), and hypothalamus (Fig. 4d).

ProSUB and SUBv are the only SUB domains that target the MPF, which also receives robust inputs from the CA1dc, CA1i, and CA1v. Additionally, ProSUB and SUBv projections are complementary: the ProSUB primarily targets the dorsal peduncular area (DP) and deep layer infralimbic cortex (ILA), whereas SUBv projects densely to superficial ILA layers and sparsely to ventral anterior cingulate cortex layer 6 (ACAv, Fig. 6a). The ProSUB and SUBv project topographically to the amygdala, ACB, and hypothalamus. Amygdala-projecting neurons are primarily distributed in SUBv/SUBvv layer 3, while amygdala inputs to SUB mostly terminate within the superficial or deep half of the molecular layer (Supplementary Fig. 9a, b; except for caudal part of the anterior basolateral amygalar nucleus or BLAa, discussed later). Rabies viral tracing of the posterior basolateral amygdalar nucleus (BLAp)-projecting neurons reveals that layer 3 SUB neurons extend thick dendritic shafts into the molecular layer that appear to bifurcate after passing through the deep half of the molecular layer (as determined by PHAL-labeled BLAp fibers, Fig. 6b).

Within the ACB, SUB projections are topographically distributed such that ProSUB and SUBv (as well as the SUBvv) project to relatively distinct lateral, intermediate, and medial areas (Supplementary Fig. 9c). The CA1v contributes a more broad terminal field which overlaps the ACB area that is also innervated by both the SUBv and SUBvv. Axons arising from the ProSUB and SUBv travel through the fornix and give off collateral projections to innervate multiple hypothalamic nuclei around the fornix (peri-fornical band) in contrast to the SUBdd and SUBvv (medial band, Fig. 6c). Fig. 6d shows the ProSUB/SUBv connectivity with areas of amygdala, septum, thalamus, septum, hypothalamus, and MPF for controlling defensive behavior (see Discussion).

The CA1vv-SUBvv global neural networks-The CA1vv and SUBvv are part of a brain-wide network with amygdala, hypothalamus, and basal forebrain regions that are distinct compared to the CA2-CA1dc-ProSUB and CA1i/CA1v-SUBv networks (Fig. 4d). First, the SUBvv is reciprocally connected with amygdala regions that receive accessory olfactory bulb (AOB) input (the posteromedial cortical amygdala area (COApm), posterior amygdalar nucleus (PA), and the posterodorsal and posteroventral medial amygdalar nucleus (MEApd, MEApv); Supplementary Fig. 9b). Second, SUBvv axons project to multiple hypothalamus regions along the 'medial band' including median preoptic nucleus (MPN), ventromedial hypothalamus (VMH), ventral premammillary nucleus (PMv) and multiple hypothalamic regions such as the dorsomedial hypothalamic nucleus (DMH) and anterior preoptic nuclei that target neuroendocrine neurons (Fig. 6c). Third, SUBvv axon terminal fields are primarily located in the dorsomedial ACB shell, which receives much less inputs from the SUBv (Supplementary Fig. 9c). Finally, SUBvv fibers uniquely innervate the ventral lateral septum (LSv) and principal subnucleus of bed nuclei of stria terminalis (BSTpr), whereas SUBvv, SUBv, ProSUB, and CA1v fibers converge within the interfascicular and transverse BST (BSTif/tr; Supplementary Fig. 9d). Fig. 6e summarizes the SUBvv connectivity with medial hypothalamus, amygdala, thalamus, and septum for 
control of neuroendocrine output and homeostasis (see Discussion). Overall, the unique neuroanatomical connectivity of the SUBdd/SUBdv vs. ProSUB/SUBv vs. SUBvv supports our computational clustering results as parts of three separate brain-wide networks.

\section{Global interactions of the multiple hippocampal subnetworks}

Although we have shown how hippocampal networks contribute to three relatively independent brain-wide networks, there are also examples where hippocampal networks contribute as a whole. Previously, we described SUBdd's relationship with the RSPv as part of the medial cortico-cortical networks ${ }^{18}$. Our new data shows that all SUB subregions can influence the medial cortico-cortical network (i.e., RSPv, ACAd, ACAv, and ILA) through relays within the anterior thalamus. First, while SUBdd and SUBdv project directly to the $\mathrm{RSPv}$, the AD/AV is also positioned to relay SUBdd/SUBdv information to the RSPv. In contrast, the ProSUB, SUBv, and SUBvv do not project directly to the medial corticalcortical network but instead project to the dorsal and ventral anteromedial thalamic nuclei (AMd/AMv), IAD/IAM, and PVT/PT, which in turn projects to the dorsal anterior cingulate cortex (ACAd), ACAv, and ILA, respectively (see Fig. 7a). Through these parallel, reciprocal thalamo-cortical and thalamo-subicular connections, information from all hippocampal networks can be integrated at different nodes along the medial cortico-cortical network, which is hypothesized to transfer visual, auditory, and spatial information to the prefrontal cortex to influence attention, motor planning, and other aspect of goal-directed behavior, such as navigation and exploratory behavior $1,18,29,30$.

Second, we found that a small cluster of neurons within the caudal BLAa (BLAa.c) is positioned as a unique hub between hippocampal subnetworks and medial prefrontal cortex, PAR, and amygdala (Fig. 7b). The distribution of CA3id/CA3ic retrograde labeling and anterogradely-labeled PAR fibers uniquely identify this small cluster of BLAa.c neurons (Fig. 7b). A coinjection that was targeted to this area confirmed that the BLAa.c neurons generate broad robust projections to innervate multiple regions across the hippocampal axis and injections within multiple hippocampal structures and medial prefrontal cortex also produced retrogradely-labeled neurons in this area (Fig. 7b). Notably, BLAa.c neurons uniquely innervate the pyramidal cell layer of the CA1 and SUB directly whereas all other amygdala regions primarily project to the molecular layer (compare to Supplementary Fig. 9a). Thus, BLAa.c neurons uniquely innervate multiple hippocampal networks and are positioned as a hub to integrate information between the medial prefrontal cortex, amygdala and hippocampus, whose interactions are critical to memory consolidation ${ }^{31}$.

Third, the MM is the only hypothalamic region that receives axonal inputs from the entire SUB in a three-dimensional topographic manner (Fig. 7c). Retrograde tracer injections into anterior vs. posterior MM revealed regional and laminar specificities of MM-projecting SUB neurons. Retrograde injections into the anterior MM labeled neurons within the lateral/deep part of SUBdd layer 1 and deep layer 3 of the ProSUB, SUBv, and SUBvv (also some layer 2). In contrast, retrograde tracer injections into posterior MM labels neurons within medial/ superficial parts of SUBd layer 1 and layer 3 neurons in rostral SUBv. Injections that were limited to the ventral part of the posterior MM distinctly labeled medial SUBdv layer 1 neurons but not SUBdd neurons. Together with the pattern of anterograde innervation, our 
data provides a comprehensive view of topographic SUB projection distribution across both the rostrocaudal and dorsoventral MM axis (Fig. 7c).

Fourth, it is well documented that the rat hippocampal Ammon's horn (primarily field CA3 and CA1) generates massive descending projections to the lateral septum (LS), which in turn shares bidirectional connections with different hypothalamic neural networks mediating expression of motivated behavior ${ }^{32}$. Our data found that the hippocampal subnetworks have distinct hippocampo-septal, hippocampo-hypothalamic, and septo-hypothalamic projections that together form parallel network systems (Fig. 8a). First, LSv and ventral LSr coinjections revealed distinct sets of input/output relationships between the CA1vv/ $\mathrm{SUBvv} \rightarrow \mathrm{LSv} \rightarrow$ medial hypothalamus vs. the $\mathrm{CA} 1 \mathrm{v} / \mathrm{SUBv} \rightarrow \mathrm{LSrv} \rightarrow$ perifornical hypothalamus (Fig. 8a; also CA1dc/ProSUB $\rightarrow$ dorsal LSr $\rightarrow$ perifornical hypothalamus). Septo-hypothalamic projections are highly similar to the hippocampo-hypothalamic projection patterns (compare to Fig. 6c). Anterograde injections into each SUB region revealed a similar pattern: ProSUB and SUBv fibers differentially target a rostrocaudal band of perifornical hypothalamic regions whereas SUBvv fibers target the periventricular and medial hypothalamus, rostrocaudally (note the SUBdd and SUBdv do not appreciably innervate the septum and completely avoid the hypothalamus except for MM). Multiple retrograde tracers targeted along the peri-fornical or medial hypothalamus further revealed that hypothalamus-projecting SUB neurons are richly heterogenous across all gene expression layers with domain specificity (Fig. 8a, note SUB-septal projections similarly arise from all layers). From a systems-level perspective, SUB could directly excite hypothalamus regions via direct glutamatergic projections or inhibit hypothalamus via recruitment of GABA-ergic septo-hypothalamic projections. LS projections to the MS/NDB provide a disinhibitory 'positive' feedback loop to all levels of the hippocampal network. In addition, SUM receives robust septal input (and minor SUB input), providing an additional feedback loop to hippocampal networks downstream of hypothalamic innervation.

The SUB (or CA3 and CA1) generates sparse direct projections to the lateral hypothalamus (lateral hypothalamic area (LHA) lateral to the fornix). Instead, all CA3 domains (except $\mathrm{CA} 3 \mathrm{vv}$ ) and CA2 project topographically to the caudal LSc, which in turn, projects to the lateral band of hypothalamus, as revealed by multiple retrograde injections (Fig. 8a). In addition, hippocampal subnetworks innervate unique topographic ACB subregions (Supplementary Fig. 9c), which in turn also generate projections to the lateral hypothalamus (Fig. 8a).

Finally, the ProSUB and SUBdv are anatomically-positioned to mediate cognitive-limbic integration through their mixed input/output profiles (Fig. 8b). The ProSUB receives input from CA1dc and CA2 and is part of the 'cognitive' dorsal hippocampal network, but provides output to 'limbic' brain regions (MPF, amygdala, hypothalamus). The SUBdv appears to play a reciprocal role within the ventral 'limbic' hippocampal network: SUBdv receives 'limbic' input from CA1i and CA1v, but sends its output to the visuospatial brain areas (RSPv, POST, PRE, PAR, AD, AV, LD) that are implicated in spatial navigation. In this way, the SUBdv could influence spatial navigation based on emotionally-salient information, whereas the ProSUB could influence social behavior with regard to spatial location. In support of this hypothesis, two recent studies have shown that ProSUB neurons 
play a role in spatial working memory encoding (but not retrieval) ${ }^{33}$ and both CA1dc and ProSUB contain neurons that encode reward location ${ }^{34}$.

\section{Discussion}

The HGEA provides the first coherent anatomical and gene expression based framework for the rodent hippocampus (see Supplementary Table 7). Over the last decade, many studies have investigated gene expression within the hippocampus, but yielded different interpretations of hippocampal organization. Thompson et. al and Dong et al. both analyzed Allen Brain Atlas data and concluded that the CA3 and CA1 can be subdivided into distinct gene expression domains. Recently, CA1 subdivisions have been further supported by multiplexed seq-FISH mapping ${ }^{16,17}$, but single-cell RNA approaches (i.e. RNAseq, DivSeq) suggested a gradual transition of gene expression cell-types, rather than discrete boundaries, along the CA1 axis ${ }^{12,15}$. In relation to our findings, the HGEA appears remarkably consistent with the CA1 subdivisions determined by seq-FISH and these subdivisions are further supported by our anatomical connectivity data. An important distinction between seqFISH and single-cell RNA approaches is that seqFISH can view multiple gene expression patterns on the same histological section, whereas single-cell RNA approaches sample data from multiple sections and brains. Comparisons across multiple tissue sections introduce variations (see Supplementary Fig. 3) which could account for the gradient-like interpretation of expression. Studies which examine only one subregion (i.e. CA1, but not CA3) and/or subsample from only one rostrocaudal level are limited toward understanding the bigger picture of hippocampal organization and how gene expression boundaries change across the rostrocaudal axis.

In this study, we report evidence for strong agreement of discrete HGEA gene expression domains with unique anatomical connectivity across the hippocampus. Throughout this manuscript, we show remarkable similarity between the HGEA atlas gene expression subdivisions and anatomical labeling patterns (which are determined solely by tracer injection site size, shape, and location). Without the context of the gene expression boundaries to guide the injection site placement, it is unsurprising that previous anatomical studies concluded that hippocampal connectivity was organized as a topographic gradient. Our data shows that injection sites that are restricted to HGEA boundaries produce labeling patterns (regional and laminar-specific) that are similarly restricted to HGEA boundaries across the rostrocaudal hippocampus. This result is confirmed by anterograde and retrograde tracer injections into other brain regions outside of the hippocampus. In the case of the CA1 and SUB, the distribution of gene expression lamina defines subregions in the same way cortical layers define cortical subregions. In most cases, anatomical connectivity is defined by the gene expression lamina (i.e. SUB layer 4 projects to thalamus), however some connections are better described by subregion.

Our analysis of hippocampal connectivity revealed novel understanding of the hippocampus as a multiscale hierarchical network. Each HGEA subregion is part of small hippocampal subnetworks which, in turn, are constituent members of larger 'dorsal' and 'ventral' subnetworks. In this way, we can better understand how the broadly-defined dorsal/ventral hippocampal dichotomy (suggested by behavioral studies) could arise from the network 
connectivity of a multitude of smaller, more specific gene expression- and anatomicallyspecific subregions.

The HGEA provides a roadmap: a structural foundation for hippocampal network connectivity that implies unique functional roles for each molecular domain. Guided by the structural connectivity, functional hypotheses can be investigated by targeting and exploiting each region's distinct gene expression. In the future, functional information can be added directly to the HGEA to provide a fully comprehensive 'dynamic' hippocampal connectome. Based on our extrahippocampal analysis and evidence from the literature, we propose testable functional hypotheses for the three CA1/SUB brain-wide networks.

First, the CA1dr/SUBdd/SUBdv network plays an essential role in regulating spatial orientation and navigation. The CA1dr and SUBdd contain neuronal cell types that are consistent with a role in processing and encoding spatial orientation. The CA1dr contains the highest density of place cells ${ }^{35}$ while the SUBdd contains "boundary vector cells" 36 . Furthermore, the SUBdd is bidirectionally connected with the caudodorsal medial entorhinal cortex (ENTm), a region that contains the highest density of most spatially-sensitive grid cells ${ }^{37}$ as well as a variety of spatially-modulated neuronal cell types ${ }^{38}$. As we described, the SUBdd and SUBdv project to complementary areas within the RSPv, POST, PRE, PAR, $\mathrm{AD}, \mathrm{AV}$, and RE. All of these brain structures (except for the novel SUBdv) have been shown to contain head-direction cells ${ }^{39}$. Head-direction signals arise from vestibular input to the dorsal tegmental nucleus (DTN) and are relayed to the anterior thalamus via the LM. Together, these regions form a highly interconnected "head-direction"-sensitive neural network that is robustly interconnected with the CA1dr-SUBdd/SUBdv network (Fig. 5e). In addition, the RSPd and LD are hubs that suggest integration between the spatial network and the visual system (VIS cortices and SC). To produce locomotor behavior for spatial navigation, the visual and spatial networks are connected with both the dorsomedial caudoputamen (CP.i.dm) ${ }^{38}, \mathrm{SNr}$ (goal-directed behavior), and SC (orientation and eye movement).

The CA2/CA1dc-ProSUB and CA1i/CA1v-SUBv intrahippocampal subnetworks are constituent parts of a much larger and complex brain-wide network in guiding social behavior (particularly aggression/defensive behavior; Fig. 6d). For example, the CA2 and CA1v have both been shown to be critical for social memory and a putative site for social memory engrams ${ }^{40,41}$. In addition, the ProSUB and SUBv target complementary parts of the amygdala (i.e., the BLAa and BLAp) and the perifornical hypothalamic nuclei (i.e., AHN and PMd; Fig. 6b,c, Supplementary Fig. 9a,b) which play a role in fear memory and defensive behaviors $42,43,44,45$. The PMd generates dense projections back to the AMv which is involved with processing contextual fear memories to predatory threats ${ }^{46,47}$ and is bidirectionally connected with the ProSUB.

Finally, the most distinguished feature of the CA1vv-SUBvv network are robust projections that directly innervate neuroendocrine-related medial hypothalamic areas and other forebrain structures in regulation of homeostasis, metabolism, and sexual behavior. In contrast to the ProSUB and SUBv, SUBvv axons robustly innervate hypothalamic nuclei along a medial rostrocaudal band, including the periventricular zone, which contains multiple 
neuroendocrine cell types (Fig. 6c) ${ }^{48}$. Furthermore, the SUBvv innervates the anteromedial BST nucleus (BSTam, Supplementary Fig. 9d) and several densely interconnected hypothalamic nuclei that target the PVH, namely the anterodorsal preoptic (ADP), anteroventral preoptic (AVP), anteroventral periventricular (AVPV), parastrial (PS) and DMH (Fig. 6c). These interconnected hypothalamic structures have been hypothesized as a hypothalamic visceral motor pattern generator network that coordinates neuroendocrine and autonomic activities in maintaining homeostasis ${ }^{49}$. In addition, the SUBvv is robustly connected to the paraventricular (PVT) thalamic nucleus which receives hypothalamic inputs and viscerosensory and pain-related information from brainstem structures (i.e., nucleus solitary tract and parabrachial nucleus) ${ }^{50}$.

\section{Hypothesis for cognitive-limbic integration}

Although the SUBdd and SUBv/SUBvv generally fit the classic view of a distinct dorsal/ ventral functional dichotomy, the ProSUB and SUBdv provide anatomical substrates for cognitive/limbic cross-over function through their extrahippocampal output (Fig. 8b). The ProSUB is a region that receives input from cognitive-related dorsal hippocampal circuits, but has extrinsic projections to limbic areas (similar to SUBv). Conversely, the SUBdv receives input from ventral hippocampal circuits, but extrinsically projects to cognitive areas in the visuospatial network (similar to SUBdd). This suggests the ProSUB may use spatial information to influence hypothalamic-mediated social behaviors, whereas the SUBdv may use emotionally-salient information for environmental navigation via its projections to RSPv, POST/PRE/PAR, AD, AV, etc.

\section{Conclusion}

In conclusion, we have shown that the hippocampus can be divided into multiple subregions with distinct combinatorial gene expression patterns and connectivity. The multiple hippocampal subregions are wired together as a multiscale hierarchical network that provides output to three brain-wide networks related to spatial navigation, social, and homeostatic/reproductive behaviors. Ultimately, the HGEA provides an anatomical and gene expression roadmap for the future functional dissection of the hippocampus.

\section{Supplementary Material}

Refer to Web version on PubMed Central for supplementary material.

\section{Acknowledgements}

This manuscript is dedicated in memory of Sigmund J. Bienkowski. This work was supported by NIH/NIMH R01 MH094360-01A1 (H.W.D), NIH/NCI U01 CA198932-01 (H.W.D), NIH/NIMH RF1 MH114112-01 (H.W.D), NIH/NIMH U01 MH114829-01 (H.W.D), NIH/NIMH F32 MH107071-01-A1 (M.S.B), and a Pilot fund (HWD) of ADRC center grant P50-AG05142. The authors would like to thank Drs. Arthur W Toga, Larry W Swanson, and Harvey Karten for advising the Mouse Connectome Project.

\section{References}

1. Fanselow MS \& Dong H-W Are the dorsal and ventral hippocampus functionally distinct structures? Neuron 65, 7-19 (2010). [PubMed: 20152109] 
2. Maruszak A \& Thuret S Why looking at the whole hippocampus is not enough-a critical role for anteroposterior axis, subfield and activation analyses to enhance predictive value of hippocampal changes for Alzheimer's disease diagnosis. Frontiers in Cellular Neuroscience 8 (2014).

3. Moser MB \& Moser EI Functional differentiation in the hippocampus. Hippocampus 8, 608-619 (1998). [PubMed: 9882018]

4. Poppenk J, Evensmoen HR, Moscovitch M \& Nadel L Long-axis specialization of the human hippocampus. Trends in cognitive sciences 17, 230-240 (2013). [PubMed: 23597720]

5. Risold P \& Swanson L Structural evidence for functional domains in the rat hippocampus. Science 272, 1484 (1996). [PubMed: 8633241]

6. Swanson L \& Cowan W An autoradiographic study of the organization of the efferet connections of the hippocampal formation in the rat. Journal of Comparative Neurology 172, 49-84 (1977). [PubMed: 65364]

7. Strange BA, Witter MP, Lein ES \& Moser EI Functional organization of the hippocampal longitudinal axis. Nature Reviews Neuroscience 15, 655-669 (2014). [PubMed: 25234264]

8. Amaral DG Emerging principles of intrinsic hippocampal organization. Current opinion in neurobiology 3, 225-229 (1993). [PubMed: 8390320]

9. Dong H-W, Swanson LW, Chen L, Fanselow MS \& Toga AW Genomic-anatomic evidence for distinct functional domains in hippocampal field CA1. Proceedings of the National Academy of Sciences 106, 11794-11799 (2009).

10. Thompson CL et al. Genomic anatomy of the hippocampus. Neuron 60, 1010-1021 (2008). [PubMed: 19109908]

11. Zeisel A et al. Cell types in the mouse cortex and hippocampus revealed by single-cell RNA-seq. Science 347, 1138-1142 (2015). [PubMed: 25700174]

12. Cembrowski MS, Wang L, Sugino K, Shields BC \& Spruston N Hipposeq: a comprehensive RNAseq database of gene expression in hippocampal principal neurons. eLife 5, e14997 (2016). [PubMed: 27113915]

13. Habib N et al. Div-Seq: Single-nucleus RNA-Seq reveals dynamics of rare adult newborn neurons. Science 353, 925-928 (2016). [PubMed: 27471252]

14. Lein ES, Callaway EM, Albright TD \& Gage FH Redefining the boundaries of the hippocampal CA2 subfield in the mouse using gene expression and 3-dimensional reconstruction. Journal of Comparative Neurology 485, 1-10 (2005). [PubMed: 15776443]

15. Cembrowski MS \& Spruston N Integrating results across methodologies is essential for producing robust neuronal taxonomies. Neuron 94, 747-751. e741 (2017). [PubMed: 28521129]

16. Shah S, Lubeck E, Zhou W \& Cai L In situ transcription profiling of single cells reveals spatial organization of cells in the mouse hippocampus. Neuron 92, 342-357 (2016). [PubMed: 27764670]

17. Shah S, Lubeck E, Zhou W \& Cai L seqFISH accurately detects transcripts in single cells and reveals robust spatial organization in the hippocampus. Neuron 94, 752-758. e751 (2017). [PubMed: 28521130]

18. Zingg B et al. Neural networks of the mouse neocortex. Cell 156, 1096-1111 (2014). [PubMed: 24581503]

19. Oh SW et al. A mesoscale connectome of the mouse brain. Nature 508, 207 (2014). [PubMed: 24695228]

20. Dong HW The Allen reference atlas: A digital color brain atlas of the C57B1/6J male mouse. (John Wiley \& Sons Inc, 2008).

21. Saunders A et al. A Single-Cell Atlas of Cell Types, States, and Other Transcriptional Patterns from Nine Regions of the Adult Mouse Brain. bioRxiv, 299081 (2018).

22. Scharfman HE The enigmatic mossy cell of the dentate gyrus. Nature reviews. Neuroscience 17, 562 (2016). [PubMed: 27466143]

23. Lorente de Nó R Studies on the structure of the cerebral cortex. II. Continuation of the study of the ammonic system. Journal für Psychologie und Neurologie (1934). 
24. Cui Z, Gerfen CR \& Young WS Hypothalamic and other connections with dorsal CA2 area of the mouse hippocampus. Journal of Comparative Neurology 521, 1844-1866 (2013). [PubMed: 23172108]

25. Dudek SM, Alexander GM \& Farris S Rediscovering area CA2: unique properties and functions. Nature Reviews Neuroscience 17, 89-102 (2016). [PubMed: 26806628]

26. Kohara $\mathrm{K}$ et al. Cell type-specific genetic and optogenetic tools reveal hippocampal CA2 circuits. Nature neuroscience 17, 269-279 (2014). [PubMed: 24336151]

27. Sik A, Penttonen M, Ylinen A \& Buzsáki G Hippocampal CA1 interneurons: an in vivo intracellular labeling study. The Journal of neuroscience 15, 6651-6665 (1995). [PubMed: 7472426]

28. Ding SL Comparative anatomy of the prosubiculum, subiculum, presubiculum, postsubiculum, and parasubiculum in human, monkey, and rodent. Journal of Comparative Neurology 521, 4145-4162 (2013). [PubMed: 23839777]

29. Vann SD, Aggleton JP \& Maguire EA What does the retrosplenial cortex do? Nature Reviews Neuroscience 10, 792-802 (2009). [PubMed: 19812579]

30. Weible AP Remembering to attend: the anterior cingulate cortex and remote memory. Behavioural brain research 245, 63-75 (2013). [PubMed: 23453992]

31. Kitamura $\mathrm{T}$ et al. Engrams and circuits crucial for systems consolidation of a memory. Science 356, 73-78 (2017). [PubMed: 28386011]

32. Risold P \& Swanson L Connections of the rat lateral septal complex. Brain research reviews 24 , 115-195 (1997). [PubMed: 9385454]

33. Cembrowski MS et al. Dissociable structural and functional hippocampal outputs via distinct subiculum cell classes. Cell 173, 1280-1292. e1218 (2018). [PubMed: 29681453]

34. Gauthier JL \& Tank DW A dedicated population for reward coding in the hippocampus. Neuron (2018).

35. O'Keefe J \& Dostrovsky J The hippocampus as a spatial map. Preliminary evidence from unit activity in the freely-moving rat. Brain research 34, 171-175 (1971). [PubMed: 5124915]

36. Lever C, Burton S, Jeewajee A, O'Keefe J \& Burgess N Boundary vector cells in the subiculum of the hippocampal formation. The Journal of Neuroscience (2009).

37. Hafting T, Fyhn M, Molden S, Moser M-B \& Moser EI Microstructure of a spatial map in the entorhinal cortex. Nature 436, 801-806 (2005). [PubMed: 15965463]

38. Sargolini F et al. Conjunctive representation of position, direction, and velocity in entorhinal cortex. Science 312, 758-762 (2006). [PubMed: 16675704]

39. Taube JS The head direction signal: origins and sensory-motor integration. Annu. Rev. Neurosci. 30, 181-207 (2007). [PubMed: 17341158]

40. Okuyama T, Kitamura T, Roy DS, Itohara S \& Tonegawa S Ventral CA1 neurons store social memory. Science 353, 1536-1541 (2016). [PubMed: 27708103]

41. Hitti FL \& Siegelbaum SA The hippocampal CA2 region is essential for social memory. Nature 508, 88-92 (2014). [PubMed: 24572357]

42. Fanselow MS \& Poulos AM The neuroscience of mammalian associative learning. Annu. Rev. Psychol. 56, 207-234 (2005). [PubMed: 15709934]

43. Tovote P, Fadok JP \& Lüthi A Neuronal circuits for fear and anxiety. Nature Reviews Neuroscience 16, 317-331 (2015). [PubMed: 25991441]

44. Swanson LW Cerebral hemisphere regulation of motivated behavior. Brain research 886, 113-164 (2000). [PubMed: 11119693]

45. Canteras NS, Pavesi E \& Carobrez AP Olfactory instruction for fear: neural system analysis. Frontiers in neuroscience 9 (2015).

46. Gross CT \& Canteras NS The many paths to fear. Nature Reviews Neuroscience 13, 651-658 (2012). [PubMed: 22850830]

47. de Lima MAX, Baldo MVC \& Canteras NS A role for the anteromedial thalamic nucleus in the acquisition of contextual fear memory to predatory threats. Brain Structure and Function 222, 113-129 (2017). [PubMed: 26951288] 
48. Biag J et al. Cyto-and chemoarchitecture of the hypothalamic paraventricular nucleus in the C57BL/6J male mouse: A study of immunostaining and multiple fluorescent tract tracing. Journal of Comparative Neurology 520, 6-33 (2012). [PubMed: 21674499]

49. Thompson RH \& Swanson LW Structural characterization of a hypothalamic visceromotor pattern generator network. Brain Research Reviews 41, 153-202 (2003). [PubMed: 12663080]

50. Li S \& Kirouac GJ Sources of inputs to the anterior and posterior aspects of the paraventricular nucleus of the thalamus. Brain Structure and Function 217, 257-273 (2012). [PubMed: 22086160] 


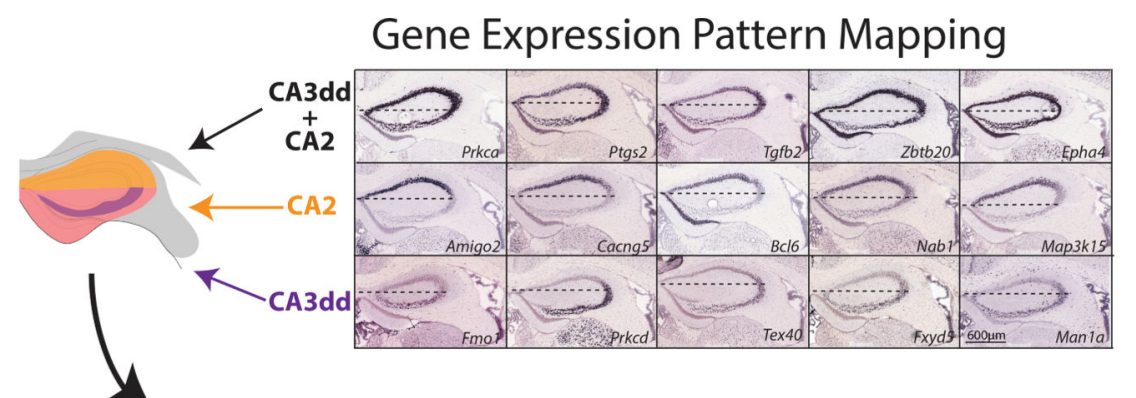

\section{Hippocampus Gene Expression Atlas (HGEA)}

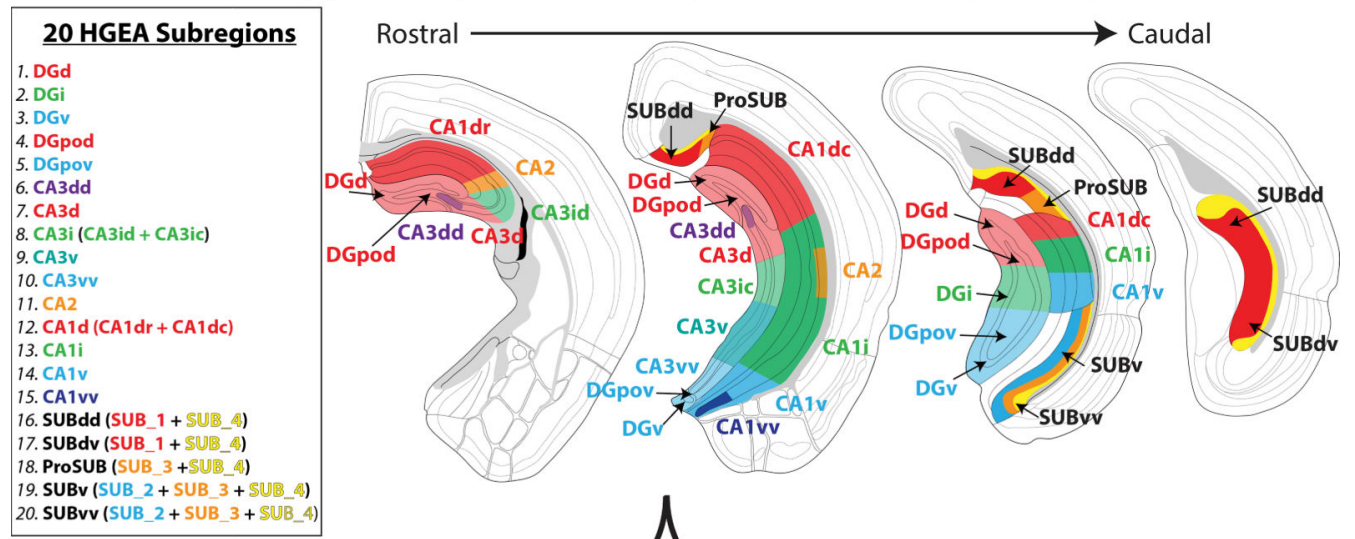

Hippocampus

Connectivity Mapping

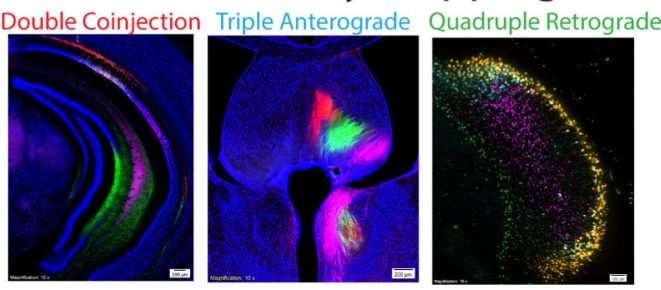

MCP iConnectome Viewer

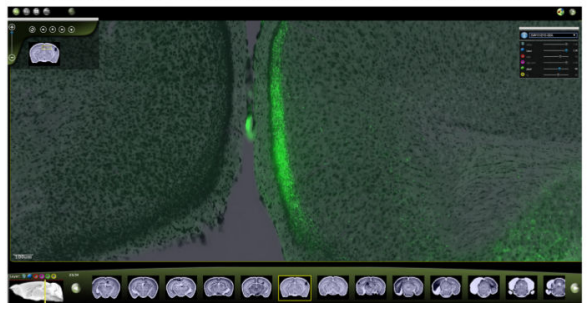

Computational Network Analysis

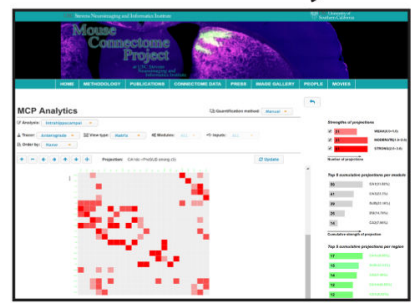

3-D Atlas Viewer

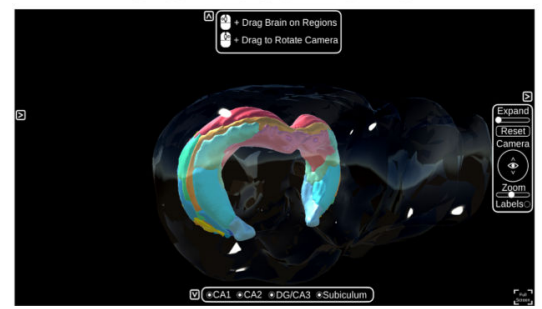

Figure 1. Experimental workflow.

(top) HGEA subregions were defined and mapped by the consensus of multiple gene expression patterns (scale bar for all images shown in bottom right panel). As an example, 5 gene expression distributions are shown that contrast CA3dd (bottom row) vs. CA2 (middle row) as well as 5 genes that are expressed in both CA3dd and CA2 (top row). Horizontal dashed line in each image corresponds to the division of CA2 genes vs. CA3dd genes which corresponds with the mapped boundary of the CA3dd/CA2 border at HGEA level 67. Consensus subregion boundaries were mapped in a similar fashion for DG, CA3, CA2, CA1, 
and SUB at all rostrocaudal levels of the hippocampus (four representative levels shown middle). Following the creation of the atlas, we examined the connectivity of each HGEA subregion as part of the Mouse Connectome Project (MCP, www.MouseConnectome.org). We used combinations of multiple retrograde and anterograde tracers in a variety of experimental strategies (double coinjection, triple anterograde, quadruple retrograde). As a resource, we have made all data openly available online at our MCP website (www.MouseConnectome.org; including connectivity image data, HGEA atlas sections, annotation tables, network analyses, and 3-D visualization tools (in situ hybridization image data can also be found at Allen Institute's website www.brain-map.org). 
a

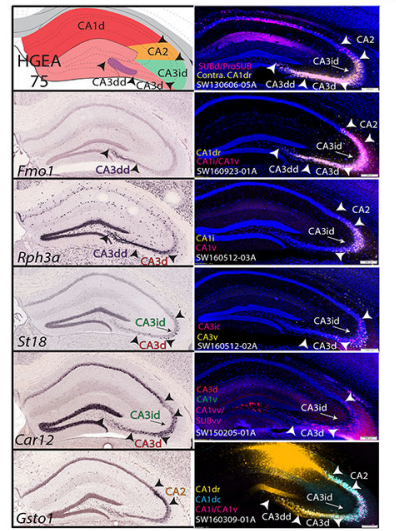

b
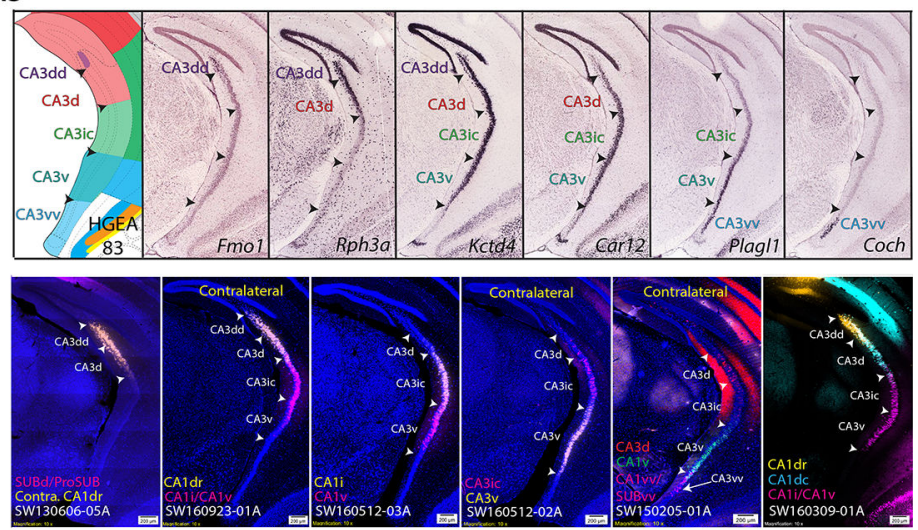

C

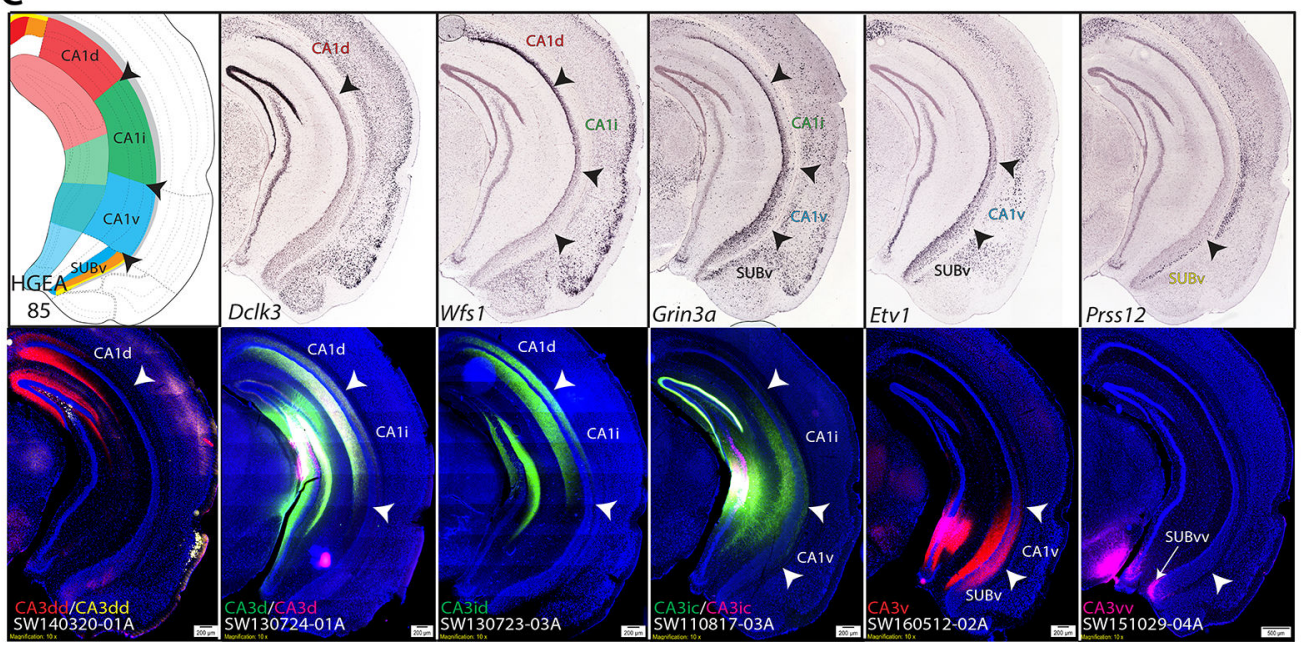

d

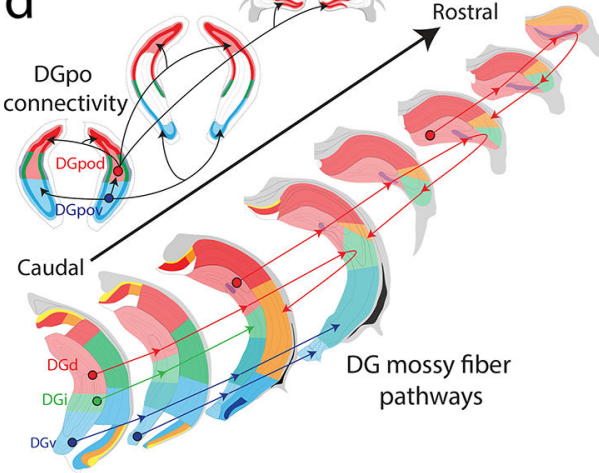

e

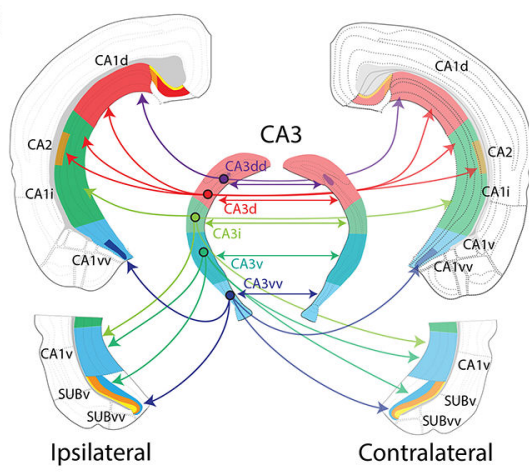

Figure 2. CA3 and CA1 gene expression and anatomical labeling patterns match closely with HGEA delineation.

For each image of tracer labeling, tracer injection sites and corresponding experimental case numbers (i.e., SW130606-05A in a) are indicated on the left-bottom (i.e., ProSUB/SUBdd or contra CA1dr). (a) Rostral hippocampus gene expression patterns and corresponding HGEA atlas section are shown on the left $(F m o 1=$ CA3dd, $R p h 3 a=$ CA3dd + CA3d, $S t 18=$ CA3id, Car12 = CA3d +CA3id, Gsto1 = CA2) while multiple retrograde labeling patterns from combinations of $\mathrm{CA} 3$ and $\mathrm{CA} 1$ subregions are shown on the right. Arrowheads demarcate HGEA subregion boundaries across the images. Retrograde injections into 
different combinations of $\mathrm{CA} 3$ and $\mathrm{CA} 1$ regions produced bilateral $\mathrm{CA} 3$ retrograde labeling patterns that appear consistent with gene expression-defined HGEA subregion boundaries. Retrograde tracer injection into the contralateral CA1dr (SW130606-05A) retrogradely labeled neurons within the CA3dd, CA3d, and CA3id (yellow) adjacent to CTB labeling (magenta) in the rostral CA2 [from ipsilateral ProSUB (some leakage into SUBdd)]. Retrograde injection into the CA1dr (SW160923-01A) shows a distinct set of labeling within the CA3dd/CA3d (yellow) but overlapping labeling with CA1i/CA1v-projecting neurons in the CA2 and CA3id (magenta). Retrograde injections into the CA1i (SW16051203A) retrogradely labeled neurons within the CA3id (yellow), but no labeling from CA1v injection (magenta). Retrograde injections into the CA3ic (magenta) and CA3v (yellow; SW160512-02A) both produce restricted labeling within the ipsilateral CA3id but in segregated superficial vs. deep neuronal populations (compare to $S t 18$ gene expression). Multiple retrograde injection in CA3d (red), CA1v (green), and CA1vv/SUBvv (magenta; SW150205-01A) revealed CA3d-projecting neurons within CA3d and CA3id (compare to Car12 gene expression). Multiple retrograde tracer injections into the CA1dr (yellow), CA1dc (cyan), and CA1i/CA1v (magenta) produce overlapping labeling patterns within the $\mathrm{CA} 3$ and CA2, but with different labeling densities distinguishing each CA3 subregion. (b) More caudal CA3 levels with gene expression patterns on top ( $F m o 1=$ CA3dd, $R p h 3 a=$ $\mathrm{CA} 3 \mathrm{dd}+\mathrm{CA} 3 \mathrm{~d}, \mathrm{Kctd} 4=\mathrm{CA} 3 \mathrm{dd}+\mathrm{CA} 3 \mathrm{~d}+\mathrm{CA} 3 \mathrm{i}+\mathrm{CA} 3 \mathrm{v}$, Car $12=\mathrm{CA} 3 \mathrm{~d}+\mathrm{CA} 3 \mathrm{id}+\mathrm{CA} 3 \mathrm{v}$, Plagl1 $=\mathrm{CA} 3 \mathrm{v}+\mathrm{CA} 3 \mathrm{vv}, C o c h=\mathrm{CA} 3 \mathrm{vv}$ ) and retrograde labeling patterns on bottom (same retrograde tracing experiments as in a). In case SW130606-05A, contralateral CA1drprojecting neurons (yellow), which were present within CA3dd, CA3d, and CA3id at more rostral levels (a), continue to be located within caudal parts of CA3dd and CA3d, but not within CA3ic (compare to Rph3a gene expression at this level). Caudal levels of case SW160923-01A shows CA1dr-projecting neurons (yellow) in CA3dd and CA3d are adjacent to CA1i/CA1v-projecting neurons (magenta) in CA3ic whereas these populations were overlapped within CA3id (a). In case SW160512-02A, CA1i-projecting neurons (yellow) are located in CA3d and CA3ic whereas CA1v-projecting neurons (magenta) are located within CA3ic and CA3v. Together, cases SW130606-05A, SW160923-01A, and SW160512-02A show that CA3id and CA3ic both project to CA1i but can be distinguished by projections to CA1d and CA1v, respectively (confirmed by anterograde labeling in $\mathbf{c}$ ). Case SW160512-03A shows associational connectivity of CA3ic (magenta) and CA3v (yellow) neurons within the contralateral CA3. Note the similarity in the CA3ic/CA3v boundary produced by CA3 associational connectivity in case SW160512-02A and Schaffer collateral CA1 connectivity in SW160512-03A. Case SW150205-01A shows multiple retrograde labeling on the contralateral side after injections into CA3d (red), CA1v (green), and CA1v/SUBvv (magenta). CA3d-projecting neurons are located within CA3d and CA3ic, CA1v-projecting neurons are located within CA3ic and CA3v, and CA1vv/SUBvvprojecting neurons are located adjacent within CA3vv. Finally, SW160309-01A shows different densities of CA1dr- (yellow) and CA1dc-projecting neurons (cyan) within CA3dd and $\mathrm{CA} 3 \mathrm{~d}$ adjacent to $\mathrm{CA} 1 \mathrm{i} / \mathrm{CA} 1 \mathrm{v}$-projecting neurons (magenta) within CA3ic and CA3v. (c) Example gene expression patterns correspond to HGEA CA1 subregions and define CA1v/SUBv border $\left(\right.$ Dclk3 $=$ CA1d, $W f_{s} 1=\mathrm{CA} 1 \mathrm{~d}+\mathrm{CA} 1 \mathrm{i}$, Grin $3 a=\mathrm{CA} 1 \mathrm{i}+\mathrm{CA} 1 \mathrm{v}+\mathrm{SUBv}$, $E t v 1=\mathrm{CA} 1 \mathrm{v}+\mathrm{SUBv}, \operatorname{Prss} 12=\mathrm{SUBv}$. Anterograde labeling of CA3 projections to CA1 revealed partially-overlapped topographic CA3 fiber distribution aligns well with gene 
expression-defined HGEA CA1 subregion boundaries (additional rostrocaudal levels shown in Supplementary Fig. 8). CA3dd projects to CA1d (more dense on medial side), CA3d and CA3id both project to CA1d (more dense lateral side) and CA1i, CA3ic projects to CA1i and CA1v, CA3v projects to CA1v and SUBv, and CA3vv projects to CA1vv and SUBvv. (d) Summary schematic of DGpo and DG mossy fiber connectivity organization (see Supplementary Fig. 5 for data). DGpod neurons (red) project bilaterally to the inner-third of the DG molecular layer across the rostrocaudal extent of the DGd and DGi as well as directly targeting the contralateral DGpod. DGpov neurons (blue) produce a similar pattern, but instead target the DGv and DGi. Below, DGd granule cell mossy fiber pathways are unique compared to the DGi and DGv. DGd mossy fibers (red) innervate DGpo cells locally before extending rostrally through the CA3 up to $1 \mathrm{~mm}$ before turning caudal to target a topographic part of CA2. The overall shape of this hairpin-like connectivity motif is different between neurons in the caudal vs. rostral parts of DGd. In contrast, DGi (green) and DGv (blue) mossy fibers extend rostrally through DGpo and CA3 in a straight-line pattern. (e) Summary schematic of CA3 subregion bilateral associational and Schaffer collateral projections to CA1. CA3dd (purple) projects to medial parts of CA1d, CA3d (red) projects to lateral parts of CA1d, CA2, and CA1i, CA3ic (green) projects to CA1i and $\mathrm{CA} 1 \mathrm{v}, \mathrm{CA} 3 \mathrm{v}$ (teal) projects to CA1v and SUBv, and CA3vv (blue) projects to CA1vv and SUBvv. For the number of tracer experiments and cross-validated results, see the Supplementary Methods. 
a

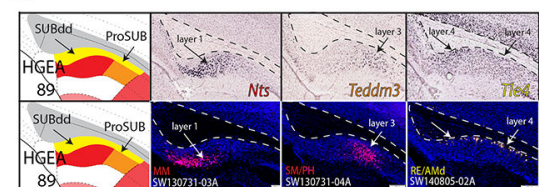

b

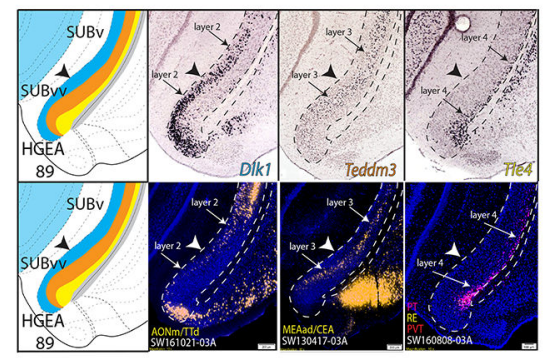

d

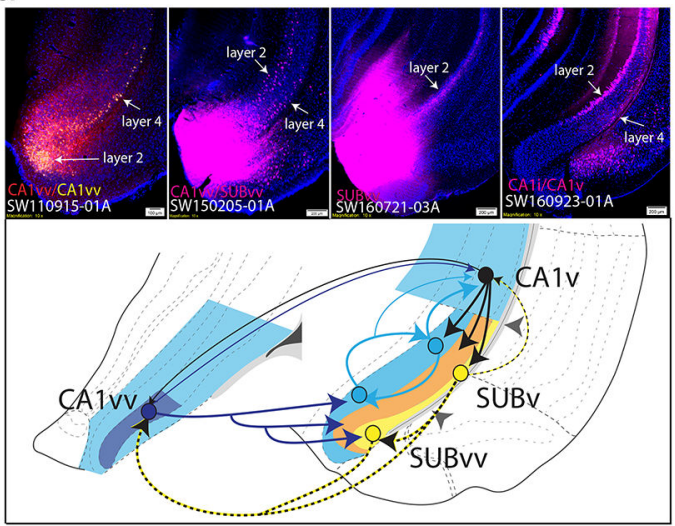

f

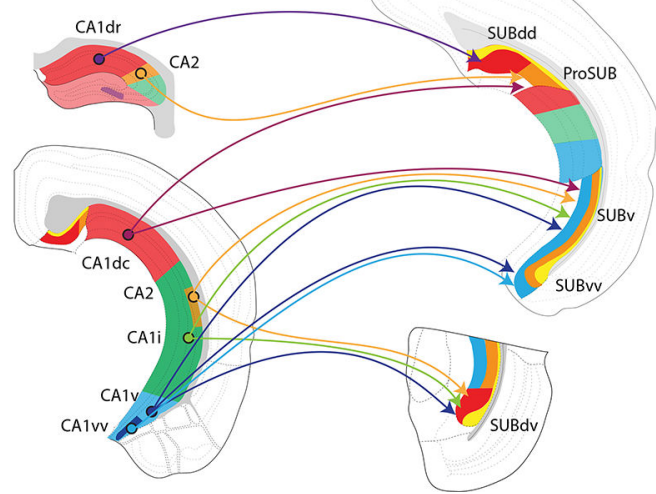

C

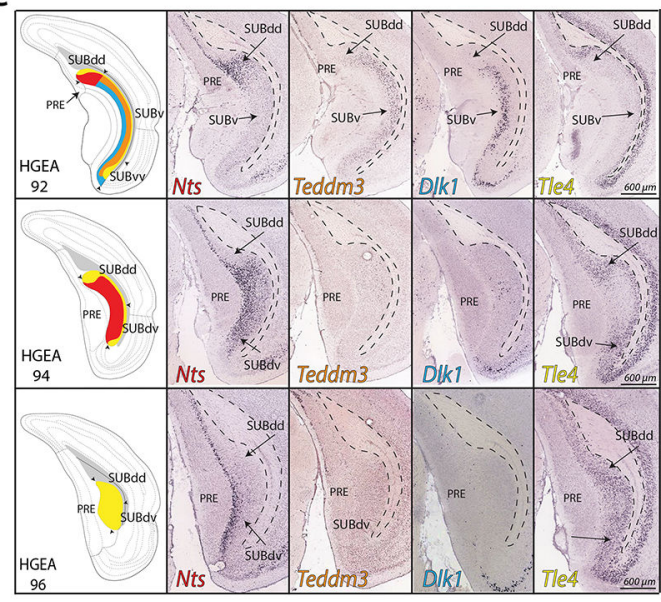

e

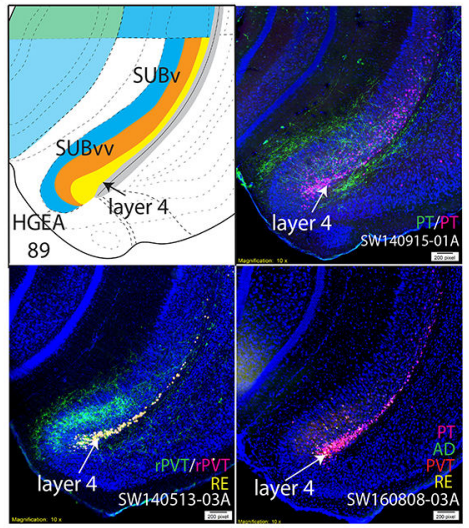

9
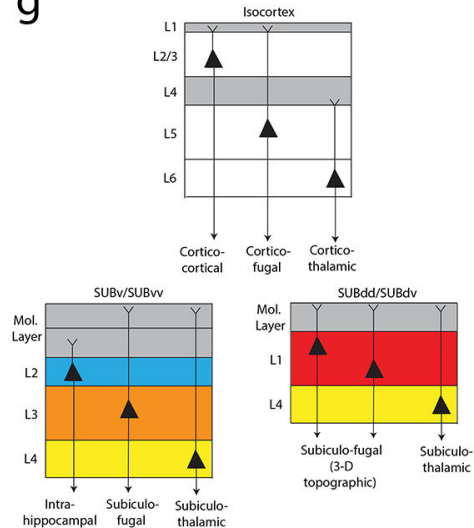

Figure 3. SUB gene expression and anatomical labeling patterns produce similar laminar organization

(a) Gene expression patterns of the classic dorsal subiculum define laminar and subregional organization that correspond to anatomical connectivity patterns. Nts and Teddm3 expression demarcate HGEA layers 1 and 3, respectively, whereas Tle4 expression identifies the deeper HGEA layer 4 adjacent to the alveus. Below, example laminar retrograde labeling patterns of MM- (SW130731-03A), SM/PH- (SW130731-04A), and RE/AMd-projecting neurons (SW140805-02A) distinctly relate to layers 1, 3, and 4, respectively. (b) Teddm3 
and Tle4 (layer 3 and 4) expression continues into the 'classic' ventral SUB, but both are located deeper to another layer identified by Dlk1 expression (layer 2). Note that there is a thickening of layer 4 at the dorsal and ventral ends of subiculum that we refer to as the dorsal and ventral 'bulbs', respectively. Below, example laminar retrograde labeling patterns of AONm/TTd- (SW161021-03A), MEAad/CEA- (SW130417-03A), and multiple thalamic-projecting neuronal cell types (PT, RE, PVT; SW160808-03A) distinctly relate to layers 2, 3, and 4, respectively. (c) At caudal levels, the distribution of the gene expression layers changes following the disappearance of the CA1. Immediately caudal to CA1 at ARA level 92, layers 2 and 3 extend dorsally to border layer 1. At ARA level 93, a ventral region containing layer 1 appears adjacent to the PAR (see Supplementary Fig. 1) and layer 1 becomes continuous at ARA level 94 following the emergence of the presubiculum (PRE). Finally, ARA level 96 represents the caudal end of the subiculum where only gene expression layer 4 is present (Nts expression is in PRE layer 3). The combinations and distribution of these layers are used to define the five SUB subregions. Scale bars on the right panels apply to all images. (d) Tracer coinjections within ventral CA1 and SUB regions revealed complex laminar-specific interconnectivity. CA1vv coinjection (SW110915-01A) produced anterograde labeling across all SUBvv layers and retrograde labeling within SUBvv layers 2 and 4. CTB injection into CA1vv/SUBvv (SW150205-01A) produced retrograde labeling within $S U B v$ and SUBvv layer 4 and the deeper part of SUBv and SUBvv layer 2. PHAL anterograde injection into SUBvv (SW160721-03A) produced anterograde labeling that travels along $\mathrm{SUBv}$ and $\mathrm{SUBvv}$ layer 2. Retrograde injection into CA1v (SW160923-01A) produces retrograde labeling in SUBv layer 4 and superficial SUBv layer 2. Together, this data suggests that CA1v, CA1vv, SUBv, and SUBvv are bidirectionally connected through two different systems of connections mediated through layers 2 and 4 (see summary diagram below). (e) Coinjection into the paratenial thalamus (PT, SW140915-01A) produces anterograde and retrograde labeling within SUBv and SUBvv layers 3 and 4 as well as anterograde labeling in the SUBvv deep molecular layer. Coinjection into the rostral paraventricular thalamus (rPVT, SW140513-03A) and Fluorogold injection into the reuniens thalamus (RE) reveals strong retrograde labeling in SUBv and SUBvv layer 4 and anterograde labeling in SUBvv layer 3 and deep molecular layer. Multiple retrograde tracing from PT, PVT, RE, and anterodorsal thalamus (AD, SW160808-03A) produces retrograde labeling that is highly restricted to layer 4. (f) Summary schematic of CA1 and CA2 projections to the five SUB subregions. (g) General laminar organization of pyramidal neurons in the isocortex (top) compared similarly to the gene expression laminar organization of the SUBv/SUBvv and SUBdd/SUBdv pyramidal neurons. Extension of dendrites into the molecular layer is based on interpretation of rabieslabeled morphology (for example, see Figs. 5c and 6b). All in situ hybridization images in (a-c) are from Allen Institute website (www.brain-map.org). For the number of tracer experiments and cross-validated results, see the Supplementary Methods. 


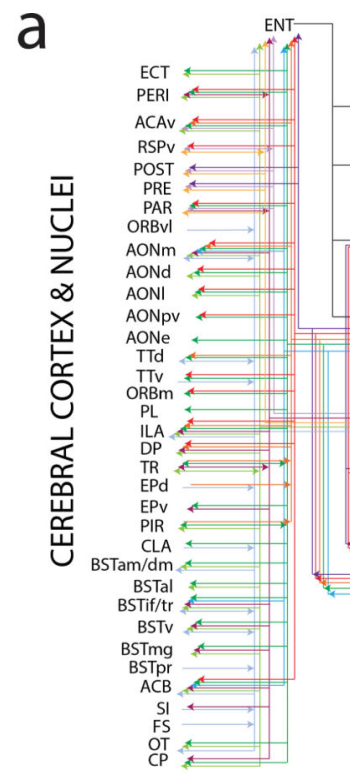

b Intra-hippocampal network 学柴al
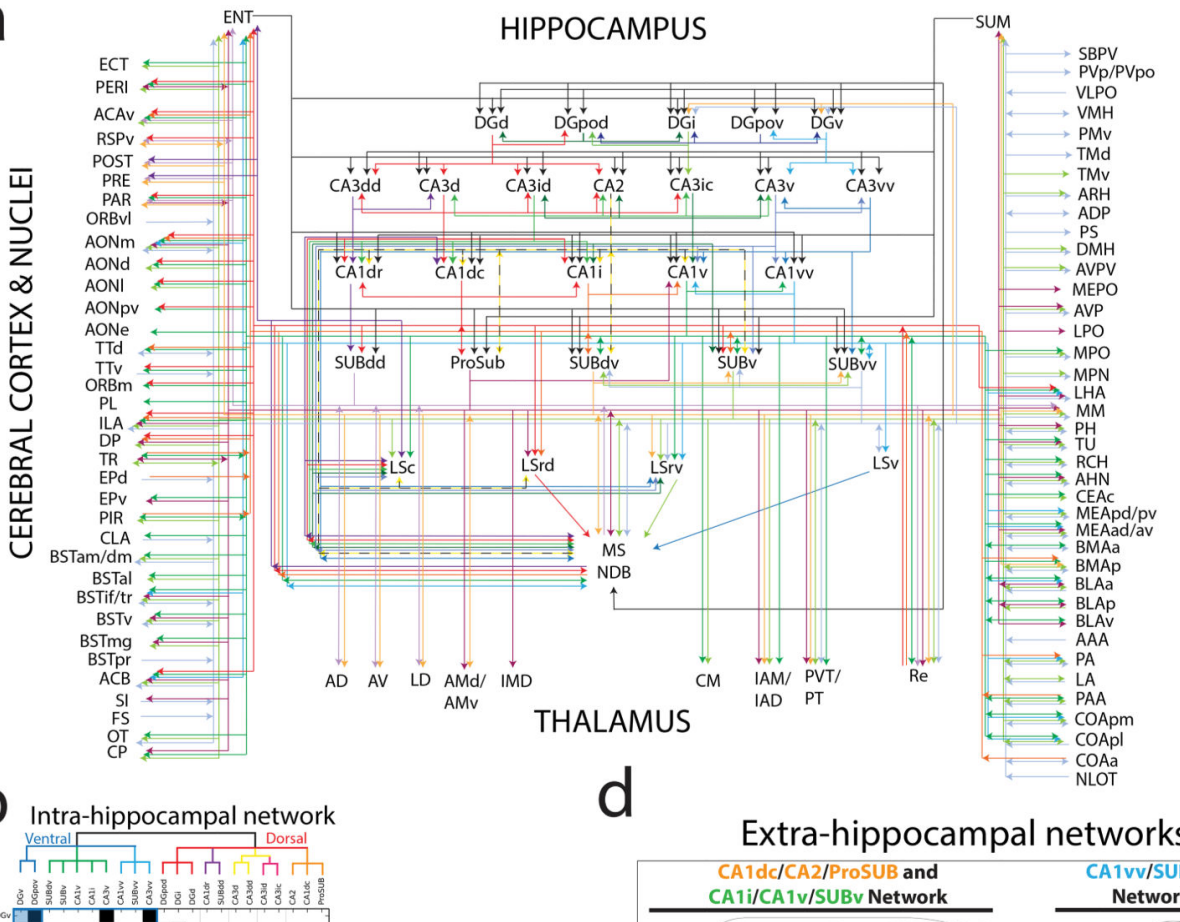

AMV

THALAMUS

CA1v , cA1vv

CA3vv

CA1i

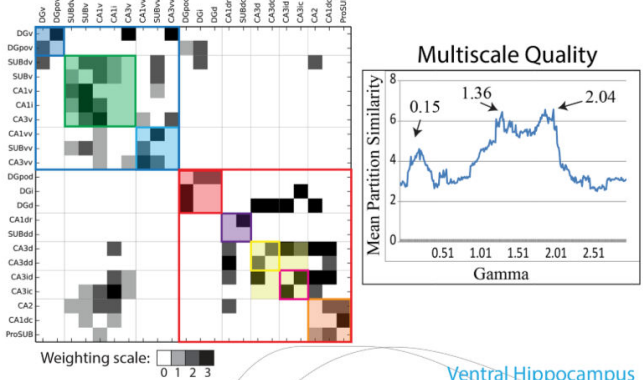

Weighting scale: $\frac{1120}{0123}$

(2)
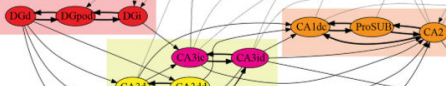

$\underset{\longrightarrow}{\longrightarrow} \longrightarrow \mathrm{CM}_{2}$

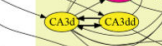

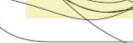

C

Extra-hippocampal networks

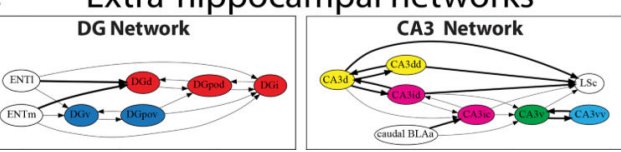

O

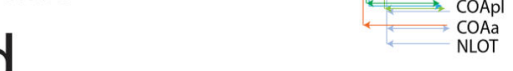

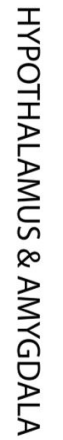

(1)

$\stackrel{\leftrightarrow}{\rightleftarrows}$ BLAp

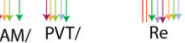

IAM/ PVT/

COApm

NOOT

Extra-hippocampal networks

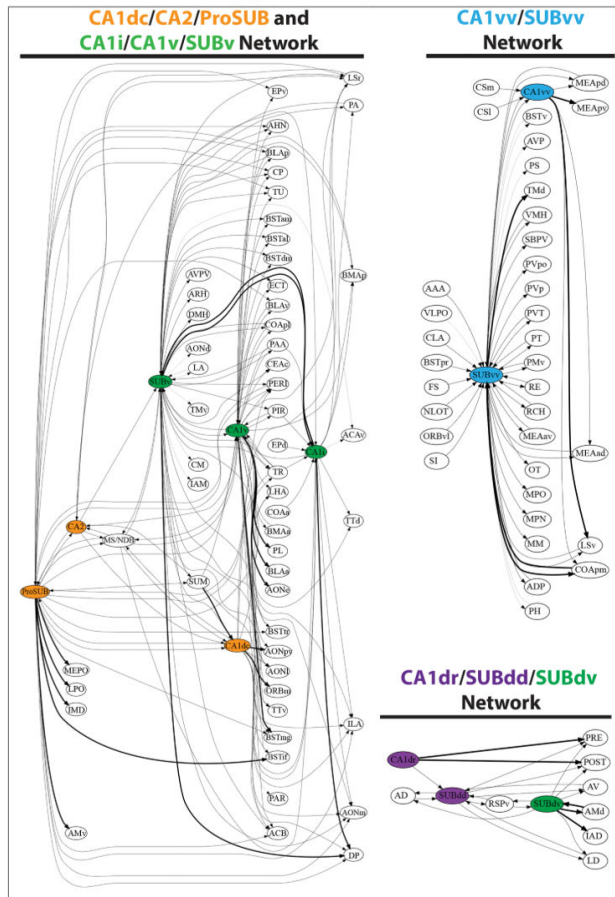

Figure 4. Multiscale neural network analysis of intra- and extra-hippocampal connections (a) Unweighted connectome wiring diagram of all inputs and outputs of HGEA hippocampal subregions. Associational connections (e.g. between CA3 subregions) are layered directly below each HGEA subregion group and extrinsic connections are layered further below. For annotated data, see Supplementary Table 5. Visit www.MouseConnectome.org for an interactive version of the wiring diagram. (b) Reordered connectivity matrix and schematic diagram showing the modular hierarchical organization of intrahippocampal subnetworks as defined by current tracing data in HGEA subregions. 
Mean partition similarity (MPS) was calculated for multiple gamma values to determine which gamma values had the highest MPS peaks $(0.15,1.36,2.04)$ to use for matrix reordering (Multiscale Quality graph). In the matrix, edges are shaded according to connectivity weight ( $0-3$, see Supplementary Table 6$)$ and colored boxes along the diagonal reflect modular communities at different scales. The large blue and red outlined boxes corresponds to the two large 'dorsal and ventral hippocampus' communities detected at 0.15 gamma, colored shaded boxes correspond to communities detected at 1.36 gamma, and smaller colored outlined boxes correspond to communities detected at 2.04 gamma. Matrix community color scheme corresponds to the organization of the schematic diagram below. In the schematic diagram, line weights refer to connectivity relationships at different scales.

Thicker lines refer to modular connections at all scales (within community) whereas thinner lines show modular relationships only at larger scales. (c and d) Five consensus brain-wide communities determined from multiscale community detection on annotated data in Supplementary Table 5 (c, DG and CA3 extrahippocampal networks; d, CA1/SUB extrahippocampal networks [CA1dc/CA2/ProSUB and CA1i/CA1v/SUBv network, CA1vv/ SUBvv network, and CA1dr/SUBdd/SUBdv network)]. Similar to the schematic in (b), line weights refer to connectivity relationships at different scales. Node coloring is maintained from intrahippocampal subnetwork analysis in $\mathbf{b}$. Different colored nodes within the extrahippocampal networks suggests multiple intrahippocampal networks provide output to a larger brain-wide network. 
a

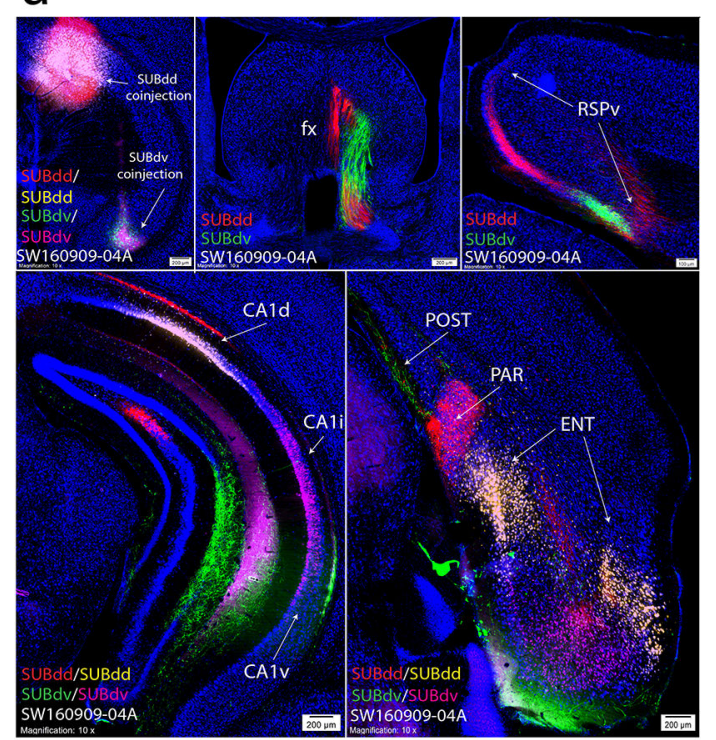

b

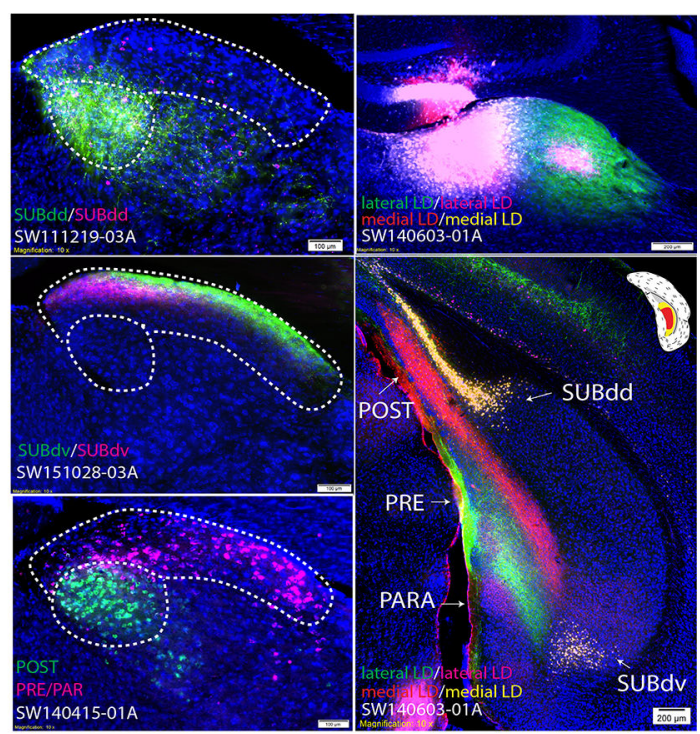

C
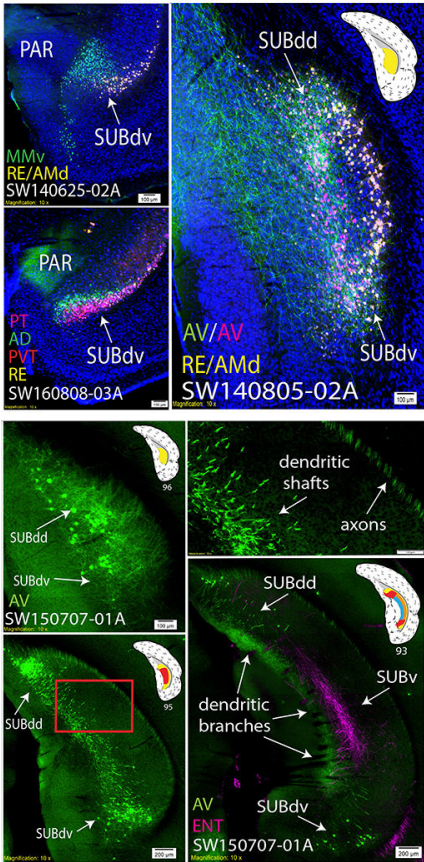

d

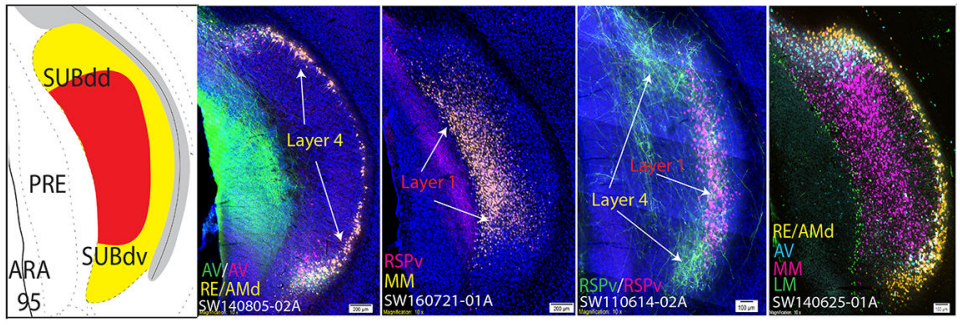

e

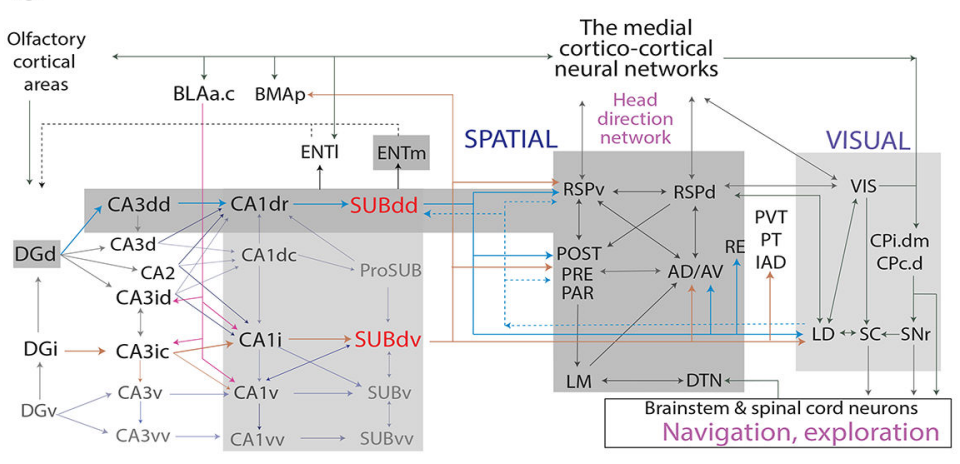

Figure 5. The role of the SUBdd and SUBdv in visuospatial integration and navigation

(a) Double coinjection experiments targeted to the SUBdd and SUBdv (SW160909-04A) reveal similar topographically-organized anterogradely labeled projections including but not limited to the fornix (middle top), RSPv (right top), and ENT (bottom right). In the hippocampus, SUBdd retrograde labeling was distributed in the CA1d, whereas SUBdv retrograde labeling was distributed adjacent within the CA1i (bottom left, highly comparable to HGEA CA1d/CA1i boundary). Both SUBdd and SUBdv project to the POST, PRE, and PAR, but have reciprocal, complementary connectivity patterns. SUBdd projects to rostral 
POST and caudal PAR while SUBdv projects to rostral PAR and caudal POST (caudal section shown in bottom right). Within the ENT cortex, SUBdd and SUBdv receive input from the lateral and intermediate bands, respectively. Anterograde and retrograde coinjection into the RSPv reveals retrograde labeling in SUBdd and SUBdv layer 1 while anterogradelylabeled RSPv fibers primarily target the dorsal and ventral ends of layer 4. (b) Anterograde and retrograde coinjections into the SUBdd (SW111219-03A) and SUBdv (SW15102803A) produces labeling pattern distributions within the LD thalamus that are highly similar to retrograde labeling patterns from the POST vs. PRE/PAR (SW140415-01A, outlined by dashed white lines). Double coinjections targeted to the medial vs. lateral part of the LD thalamus produced retrograde labeling within SUBdd and SUBdv layer 4 with laminar specific anterogradely labeled projections to the POST/PRE/PAR. (c) Organization of thalamic-projecting neurons. MM-projecting layer 1 neurons are distinct from RE/AMdprojecting layer 4 neurons within SUBdv (SW140625-02A). Within the bulbs of layer 4, AD-projecting neurons (similar to LD- and AV-projecting neurons) are distributed more superficial compared to RE-, PVT-, and PT-projecting neurons (SW160808-03A). At the caudal end of the subiculum, the AV-projecting layer 4 neurons in the dorsal and ventral bulbs join to become continuous along the medial part of the subiculum (SW140805-02A). Injection of G-deleted rabies virus into the AV thalamus (SW150707-01A) retrogradely labels AV-projecting layer 4 neuron cell bodies, axons, and dendrites. AV-projecting neurons at ARA 96 send their axons into the alveus while their thick dendritic shafts extend rostrally through the SUB at ARA 95 to bifurcate into thin dendritic branches in the superficial molecular layer at ARA 93 and avoid PHAL-labeled ENT axon fibers within the deeper molecular layer. Top right panel is magnified from red rectangle area in bottom left panel. (d) Comparison of multiple tracers reveals SUBdd and SUBdv projection cell types. In SW140805-02A, AV- and RE/AMd neurons were found to be relatively distinct projection neuron cell types within layer 4 . Note, anterogradely-labeled AV fibers also terminate specifically in the dorsal and ventral bulbs. In contrast, almost all RSPv-projecting neurons were a subset of MM-projecting neurons in layer 1 (SW160721-01A). While RSPvprojecting SUB neurons are located in layer 1, anterogradely-labeled fibers from the RSPv predominantly terminate in the layer 4 dorsal and ventral bulb regions (SW110614-02A). Finally, case SW140625-01A contains four retrograde tracer injections into the RE/AMd, $\mathrm{AV}, \mathrm{MM}$, and LM that provides a comprehensive picture of projection cell types in the SUBdd and SUBdv at ARA level 95 that is remarkably similar to the HGEA. LM-projecting neurons are located primarily in PRE layer 3, MM-projecting neurons are located primarily within layer 1, and RE/AMD and AV-projecting neurons are located primarily within layer 4. (e) Wiring schematic diagram of SUBdd and SUBdv network connections with brain regions that contribute to visuospatial behavior (see Discussion). For the number of tracer experiments and cross-validated results, see the Supplementary Methods. 


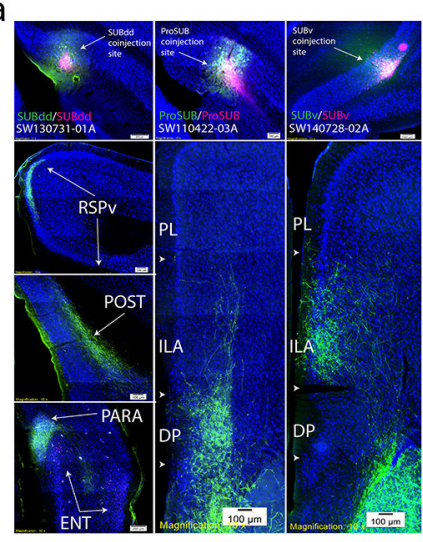

b

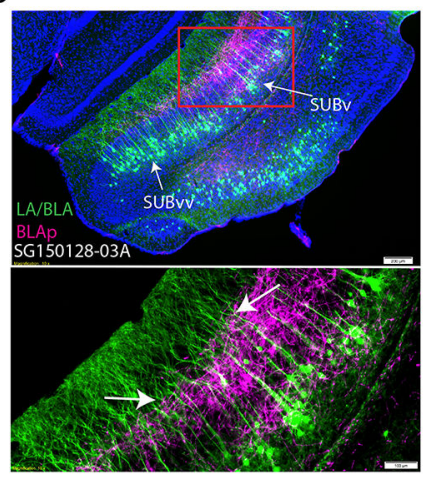

d

ProSUB and SUBv networks in controling hypothalamic defensive sysem

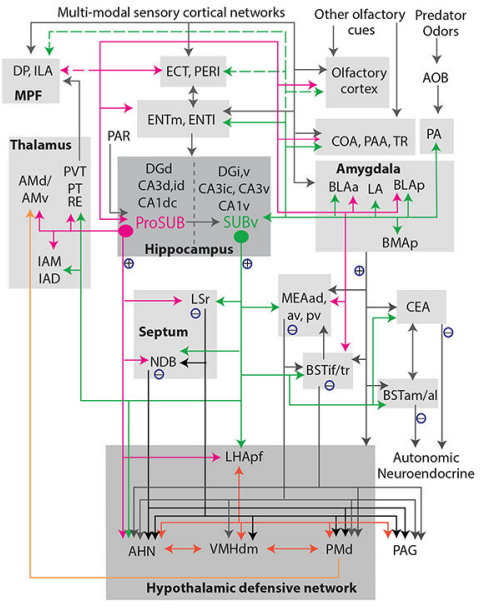

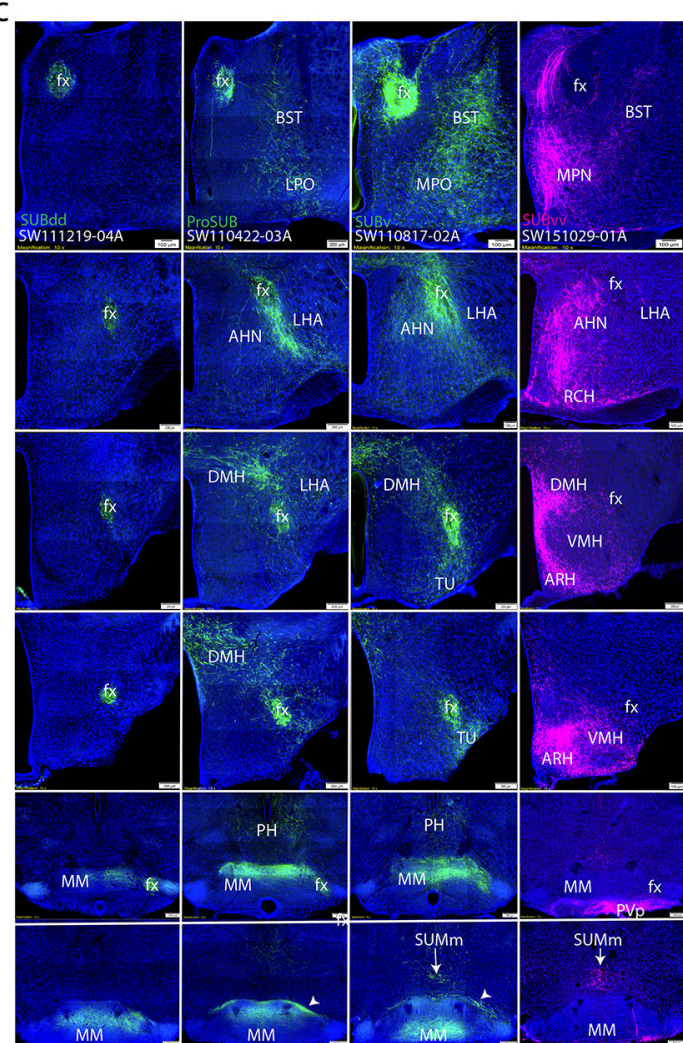

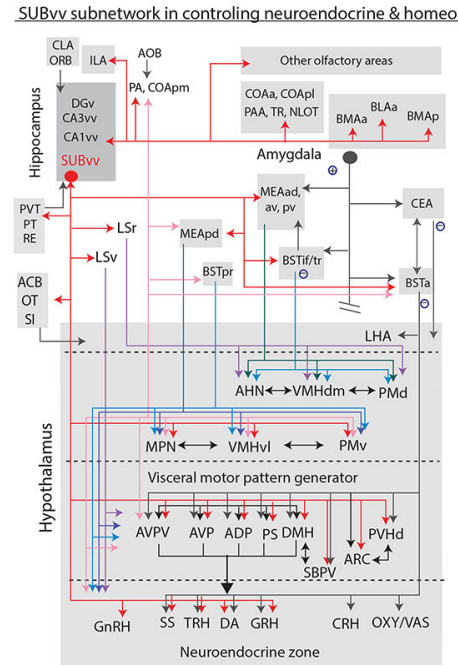

Figure 6. Distinct connectivity patterns of the ProSUB, SUBv, and SUBvv

(a) SUBdd, ProSUB, and SUBv coinjections show that while the SUBdd targets the RSPv, POST/PRE/PAR, and ENT lateral band, ProSUB and SUBv neurons target complementary parts of the MPF. Anterogradely labeled ProSUB fibers robustly innervate the DP with lighter input to ILA deep layers (SW110422-03A) while SUBv fibers densely target ILA superficial layers (SW140728-02A). (b) Subiculum projections to the amygdala arise primarily from SUBv and SUBvv layer 3 (also ProSUB) whereas amygdala fibers primarily terminate in different parts of the molecular layer (see Supplementary Figure 8a,b). Injection 
of G-deleted rabies virus into the BLAp (SG150128-03A) reveals the dendritic branches of BLAp-projecting neurons (green) among PHA-L labeled BLAp fibers (pink). Closer examination of the amygdala-projecting dendrites reveals a thick shaft that bifurcates after passing through the BLAp terminal field (arrow). (c) Subicular projections to the hypothalamus are organized along rostrocaudal bands (all image columns are organized rostral (top) to caudal (bottom)). SUBdd (and SUBdv) fibers bypass most of the hypothalamus via the fornix to innervate the MM (left column). The ProSUB and SUBv (middle columns) differentially innervate multiple hypothalamic nuclei around the fornix (perifornical band) such as the anterior hypothalamic nucleus (AHN). In contrast, SUBvv fibers (right column) target multiple hypothalamic nuclei along the periventricular and medial hypothalamus (medial band). (d and e) Schematic models showing ProSUB and SUBv connectivity with the hypothalamic defensive behavior network (d) and SUBvv connectivity with brain networks controlling metabolism, sexual behavior, and neuroendocrine function (e). For the number of tracer experiments and cross-validated results, see the Supplementary Methods. 
a

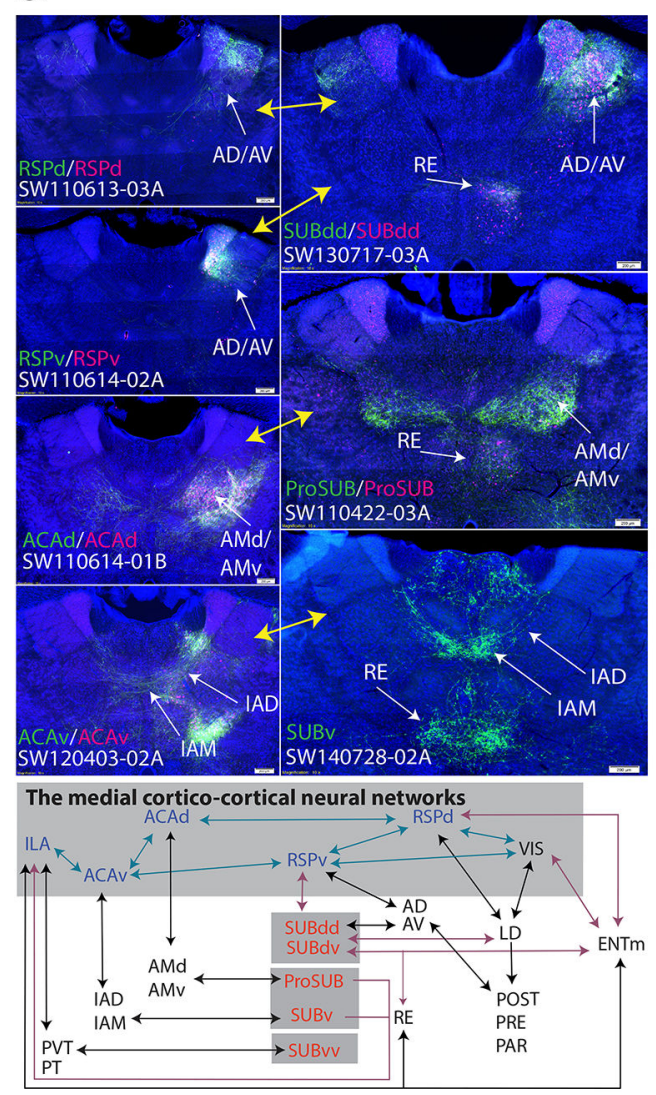

b
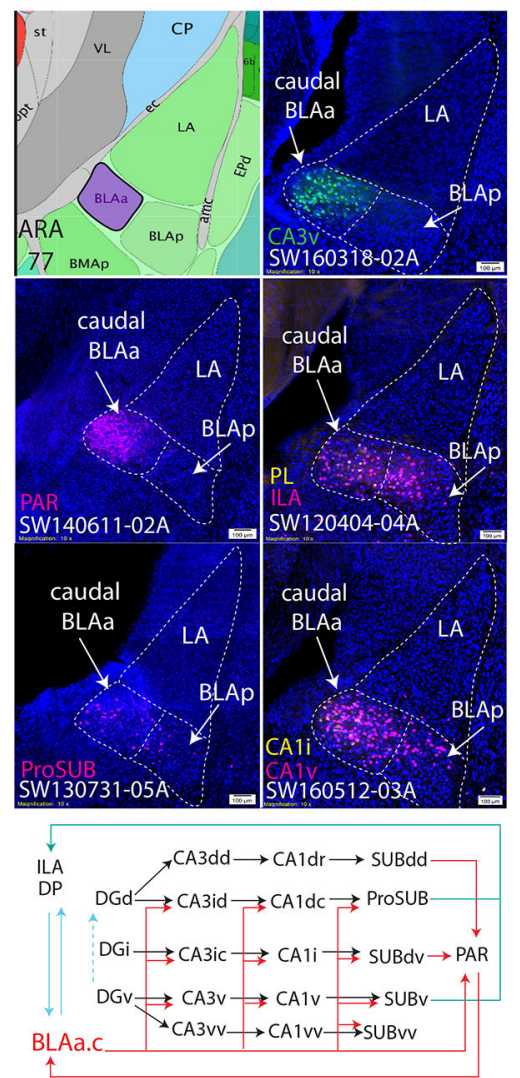
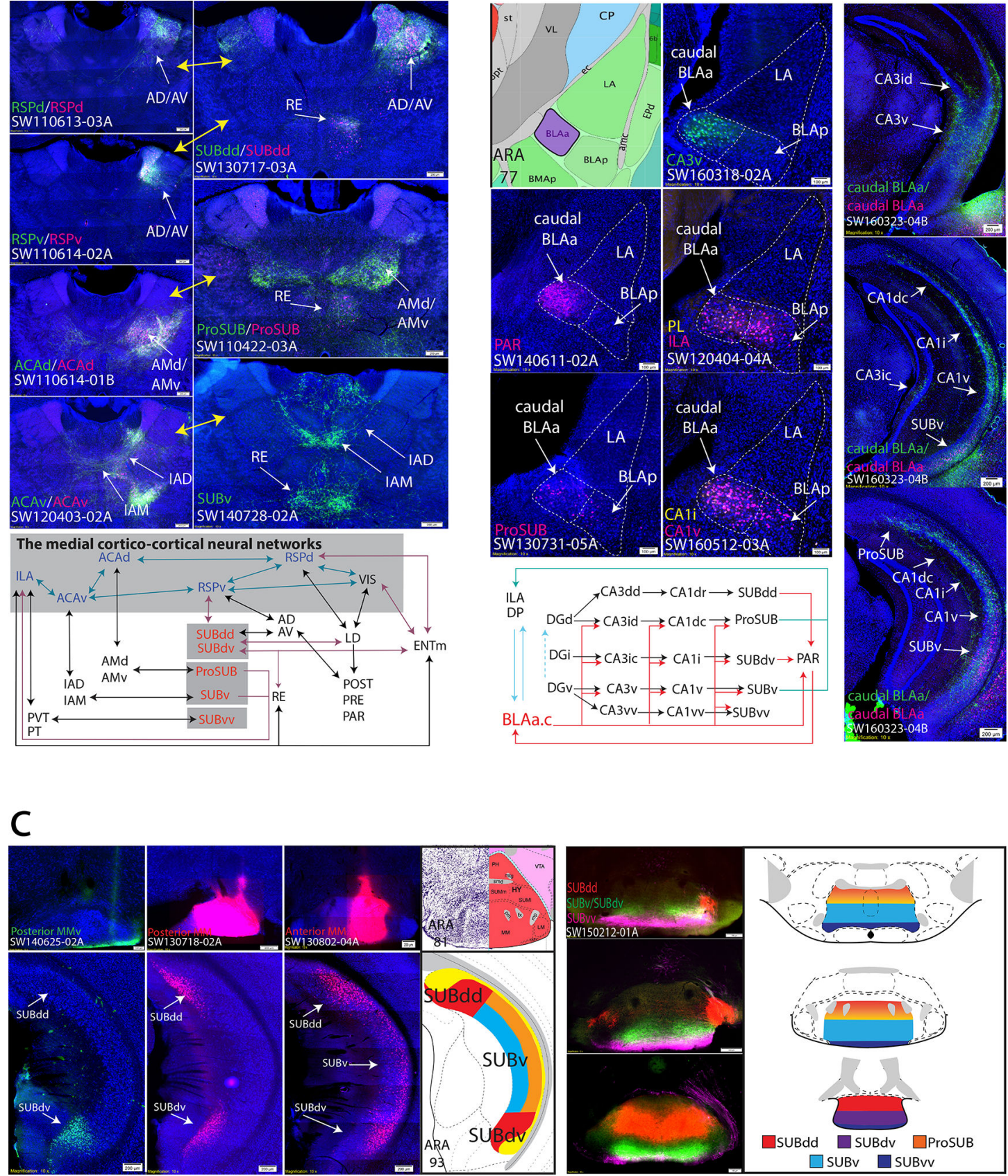

Figure 7. Brain systems with multiple interactions among hippocampal neural networks (a) Subiculum regions are topographically, bidirectionally connected with the anterior thalamic nuclei (right) in a way that is highly similar to the projection patterns observed following injections within RSP and ACA cortices (left, yellow arrows refer to similar connectivity patterns). Anterior thalamic regions serve as discrete relays for SUB information to modulate different levels of the medial cortico-cortical network (schematic below). (b) Retrograde tracer injections into the $\mathrm{CA} 3 \mathrm{v}$ specifically label a small dense cluster of neurons in the caudal BLAa (SW160318-02A) that is also specifically innervated 
by the PAR (SW140611-02A). Dashed white lines outline cytoarchitectural boundaries of the LA, caudal BLAa, and BLAp. In addition, the caudal BLAa also contains retrogradely labeled neurons following injections into many brain regions including the ProSUB, CA1i, CA1v, PL, and ILA. Double coinjection experiment targeted to the caudal BLAa (SW160323-04B) reveals a broad distribution of anterogradely-labeled fibers that, in contrast to other amygdala nuclei (see Fig. 6b), directly targets hippocampal neuron cell bodies within the CA3id, CA3ic, CA3v, ProSUB, CA1dc, CA1i, CA1v, SUBv. Together, the caudal BLAa is a unique amygdalar region positioned as a hub between multiple hippocampal networks and MPF. (c) Organization of the subiculum projections to MM. An injection isolated specifically to the ventral part of posterior MM (MMv, SW140625-02A) only labeled neurons within the SUBdv, while a larger injection into the posterior MM (SW130718-02A) specifically labeled neurons in both the medial/superficial part of SUBdd and SUBdv layer 1. Whereas posterior MM injections label superficial neurons in layer 1, injection into the anterior MM (SW130802-04A) retrogradely-labeled neurons deeper within SUBdd/SUBdv layer 1 as well as layer 3 neurons in SUBv. Triple anterograde tracer injections into the SUBdd, SUBdv/SUBv, and SUBvv (SW150212-01A) reveal bilateral fiber terminals across the rostrocaudal MM. Overall, the entire SUB topographically projects in unique termination zones across the rostrocaudal axis of the MM (summarized in the diagram on the right). For the number of tracer experiments and cross-validated results, see the Supplementary Methods. 
a
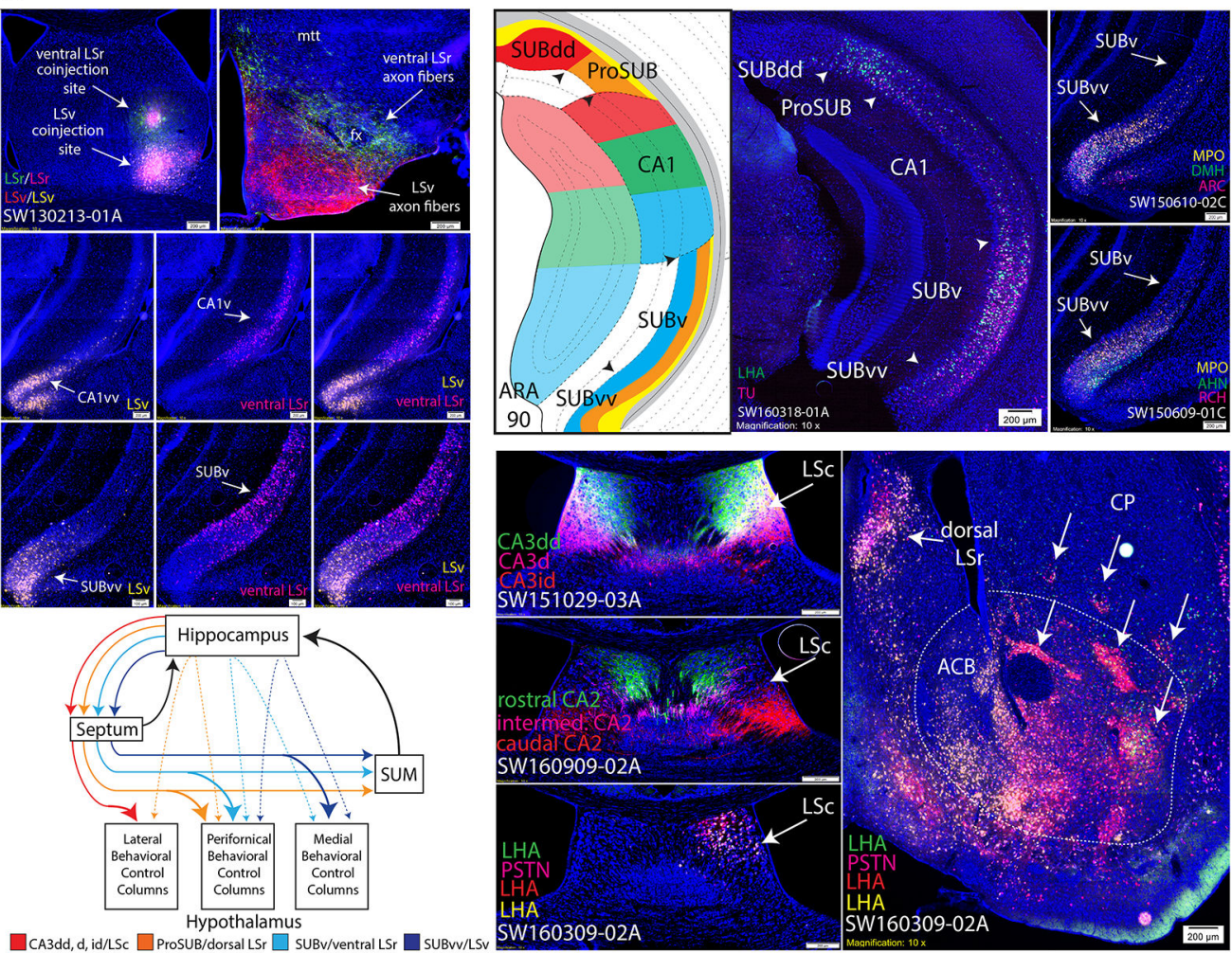

b

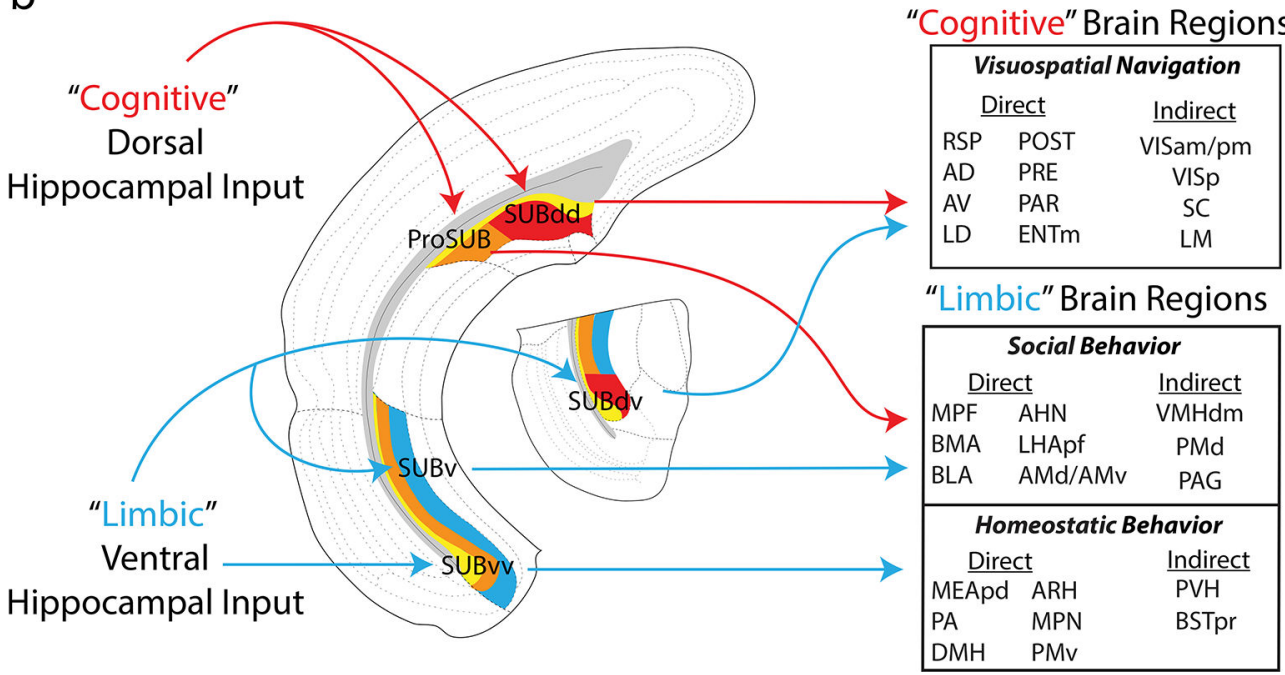

Figure 8. Hippocampal-septo-hypothalamic networks and cognitive-limbic integration through subiculum output pathways

(a) Hippocampal network output contributes to parallel septo-hypothalamic pathways. Double coinjection experiment into the ventral LSr and LSv produced retrograde labeling distinctly within all layers of the CA1v/SUBv and CA1vv/SUBvv, respectively, and anterogradely targeted perifornical and medial bands of the hypothalamus (SW130213-01A; highly similar to SUB hypothalamic projection in Fig. 6c). Multiple retrograde tracers injected along the perifornical and medial bands produces heterogenous mixture of 
retrograde labeling across all layers of ProSUB/SUBv (SW160318-01A) and SUBvv (SW150610-02C and SW150609-01C). Multiple dorsal CA3 and CA2 subregions topographically innervate the LSc (SW151029-03A and SW160909-02A) which overlaps retrograde labeling from multiple retrograde tracer injections into the lateral hypothalamic band (lateral to perifornical band, SW160309-02A). Lateral hypothalamic band (also SI) receives input from the nucleus accumbens (arrows identify striosome-like multi-labeled structures). Overall, hippocampal networks form four parallel networks that directly and indirectly (via septum) innervated broad regions of the hypothalamus for motivated behavior. (b) From a systems perspective, the 'cold cognitive' dorsal hippocampus and 'hot affective' ventral hippocampus ultimately provide input to distinct subiculum regions. However, the outputs of the ProSUB and SUBdv suggest pathways for cognitive-limbic crossover function in two ways. The ProSUB receives cognitive visuospatial information from dorsal hippocampus and then provides output to limbic regions that are similar to the output of the SUBv. In reciprocal fashion, the SUBdv receives emotionally-salient limbic information from ventral hippocampus and projects to cognitive spatial navigation brain regions similar to the SUBdd. For the number of tracer experiments and cross-validated results, see the Supplementary Methods. 